\title{
On the trait implications of social behaviors: Kindness, intelligence, goodness, and normality ratings for 400 behavior statements
}

\author{
ROBERT W. FUHRMAN \\ University of Texas at San Antonio, San Antonio, Texas \\ GALEN V. BODENHAUSEN \\ Michigan State University, East Lansing, Michigan \\ and \\ MERYL LICHTENSTEIN \\ Leo Burnett USA, Chicago, Illinois
}

\begin{abstract}
Four hundred stimulus behaviors and their mean normative ratings for kindness, intelligence, goodness, and normality were presented for use in person perception and memory studies. Each of the four normative ratings was based on a separate sample of 35 to 39 undergraduate students from the University of Illinois. Rankings of the mean ratings were provided to facilitate a quick comparison of the behavior ratings along each of the four trait dimensions. In addition, a cluster analysis of the behaviors was reported, using the mean kindness, intelligence, and normality ratings as defining variables. Six clusters were distinguished: (1) behaviors that primarily conveyed kindness; (2) behaviors that primarily conveyed unkindness; (3) behaviors that conveyed an unusual amount of intelligence; (4) behaviors that conveyed general intelligence; (5) behaviors that conveyed a general lack of intelligence; and (6) behaviors that conveyed very little information about kindness or intelligence.
\end{abstract}

Researchers of person memory and social judgment often present research participants with behavioral information about hypothetical persons in order to study the manner in which social impressions are formed and organized in memory. In such research, it is often necessary to expend a considerable amount of time and effort generating and pretesting a large number of behavior statements before conducting the actual experiment. The current study provides an organized data base of social behavior statements that will permit researchers who use such statements for research stimuli to avoid the tedium of normative pretesting.

In this article, we present evidence bearing on the trait implications of 400 common social behaviors. The specific behaviors were generated and collected over the past 6 years for the purposes of various studies of person memory and social judgment (see Fuhrman, 1987; Lichtenstein \& Srull, 1987; Srull, Lichtenstein, \& Rothbart, 1985). The behaviors were judged by undergraduates for how well they exemplify the traits of kindness and intelligence. In creating this list, we attempted to include behaviors that vary substantially in the degree to which they are relevant to these trait concepts and in the degree to which they exemplify the positive or negative aspects of the

Correspondence may be addressed to Robert W. Fuhrman, Division of Behavioral and Cultural Sciences, University of Texas at San Antonio, San Antonio, TX 78285-0652. traits. In addition, we collected goodness and normality ratings for each of the behaviors. The latter ratings are useful for the researcher who desires to control for the overall desirability or distinctiveness of the behaviors used in research studies.

\section{METHOD}

\section{Subjects}

The behavior statements were rated by 146 male and female undergraduate students from the University of Illinois at Urbana-Champaign. The participants received partial course credit in an introductory psychology class.

\section{Behavior Generation}

The behavior statements were initially generated by the authors for studies in which the behaviors were used to convey information about a single hypothetical stimulus person. The behaviors were selected for inclusion in the current study on the basis of several criteria. First, we selected behaviors principally related to the positive and negative poles of the kindness and intelligence trait dimensions. These dimensions were selected because much of the work done in the person memory domain has been concerned with impressions and social judgments based on these dimensions (cf. Srull et al., 1985). In addition, an attempt was made to select behaviors that vary in the degree to which they exemplify the positive and nega- 
tive pole of each trait dimension. Additional items were added to the list to provide instances of behaviors that were presumably irrelevant to the traits of kindness and intelligence.

We also attempted to control the length of the behavior statements. All statements were worded to fit on a single line of text in the rating booklets. This constraint was imposed to facilitate the use of these items in studies that assess reading speed and recall. Shorter items of a relatively uniform length are easier to display on a computer screen and are generally easier to score after a recall task.

\section{Procedure}

Participants received a 12-page booklet containing the list of 400 behaviors. Each page was marked with an 11-point judgment scale (with anchor terms) at the top and a list of 30 to 35 behaviors printed below the scale. The subjects were instructed to fill in the blank space after each behavior with the number from the scale that they felt was most appropriate for the behavior. They were strongly encouraged to ask the experimenter if any confusion arose concerning the behavior statements or the rating scales. Thirty-seven subjects rated the behaviors on an 11-point kindness scale ranging from 0 ("extremely unkind") to 10 ("extremely kind"). Thirty-five additional subjects rated the behaviors on an intelligence scale ranging from 0 ("extremely unintelligent") to 10 ("extremely intelligent"). Another 39 subjects rated the behaviors on a goodness scale ranging from 0 ("extremely bad") to 10 ("extremely good"). Finally, another 35 subjects rated the behaviors on a normality scale ranging from 0 ("extremely unusual") to 10 ("extremely normal"). The presentation order of the behavior statements was varied across forms to militate against order effects. It typically took 45-60 min for subjects to complete the task. They were thanked and debriefed after the completion of the ratings.

\section{RESULTS AND DISCUSSION}

The behavior statements and the mean ratings of their implications for goodness, kindness, intelligence, and normality are presented in the Appendix. The behaviors are organized in the Appendix according to the magnitude of their mean ratings along the goodness dimension (starting with the highest rating and ending with the lowest). The four mean trait ratings for each behavior are listed on each row.

\section{Behavior Rankings}

The rankings of the mean ratings for each behavior refer to the position of the mean rating of each behavior, relative to the ratings of the other behaviors along the same trait dimension. The average rank of the behaviors is computed and listed when two or more behaviors have the same mean trait rating. These behavior rankings provide a quick way for the reader to identify behaviors of similar (or different) magnitude along any one of the four trait dimensions. More generally, behaviors convey increasingly less goodness, kindness, and intelligence as they appear progressively later in the Appendix.

\section{Cluster Scores}

A cluster score for each behavior is listed in the far right-hand column of the Appendix. This score refers to the behavior cluster that each behavior was assigned on the basis of a cluster analysis performed using the mean kindness, intelligence, and normality ratings of the behavior as observed values. The mean goodness ratings were not included in this analysis because our primary concern was to distinguish behaviors that conveyed relatively more intelligence than kindness from those that conveyed relatively more kindness than intelligence. The first six clusters obtained accounted for over $82 \%$ of the variance in the ratings. Additional clusters did not contribute substantially to the amount of variance explained. The six clusters can be distinguished as follows:

Cluster 1 contains 70 behaviors that were rated very high in kindness $(M=8.02)$, relatively high in intelligence $(M=6.95)$, and moderately high in normality $(M=5.99)$. These behaviors convey information about a person's kindness and, to a lesser extent, about his or her intelligence.

Cluster 2 contains 80 behaviors that were rated very low in kindness $(M=2.23)$ and intelligence $(M=2.58)$, and moderately low in normality $(M=3.29)$. These behaviors convey information that a person is unkind and, to a slightly less degree, information about his or her lack of intelligence.

Cluster 3 contains 11 behaviors that were rated moderate in kindness $(M=5.86)$, extremely high in intelligence $(M=8.41)$, and extremely low in normality $(M=2.88)$. These behaviors convey much more information about a person's intelligence than about the person's kindness. The information also implies an abnormal amount of intelligence.

Cluster 4 contains 73 behaviors that were rated moderate in kindness $(M=5.67)$, relatively high in intelligence $(M=7.04)$, and moderate in normality $(M=5.08)$. These behaviors generally convey more information about a person's intelligence than about the person's kindness.

Cluster 5 contains 86 behaviors that were rated moderate in kindness $(M=4.34)$, low in intelligence $(M=3.30)$, and moderate in normality $(M=4.34)$. These behaviors convey information about a person's general lack of intelligence and, to a lesser extent, information about the person's unkindness.

Cluster 6 contains 80 behaviors that were rated relatively moderate in kindness $(M=5.55)$ and intelligence $(M=5.86)$, and high in normality $(M=6.69)$. These behaviors appear to be uninformative about a person's kindness or intelligence.

The cluster scores in the Appendix are useful for the researcher who is interested in selecting behaviors that convey more information about one of the trait dimensions than about another. However, the mean ratings of 
Table 1

Correlations between the Mean Trait Ratings of All $\mathbf{4 0 0}$ Behaviors

\begin{tabular}{lcccc}
\hline \multirow{2}{*}{$\begin{array}{l}\text { Normative } \\
\text { Ratings }\end{array}$} & \multicolumn{4}{c}{ Variables Correlated } \\
\cline { 2 - 5 } & 1 & 2 & 3 & 4 \\
\hline 1. Kindness & & $.76^{*}$ & $.93^{*}$ & $.46^{*}$ \\
2. Intelligence & & & $.91^{*}$ & $.37^{*}$ \\
3. Goodness & & & & $.46^{*}$ \\
4. Normality & & & &
\end{tabular}

Note $-N=400 .{ }^{*} p<.01$.

each specific behavior should also be considered in this selection process since some of the behaviors in each cluster will manifest more of the prototypical characteristics of the overall cluster than will other behaviors.

\section{Trait Rating Correlations}

The last set of results we consider pertains to the relationships observed between the four mean trait ratings across all 400 stimulus behaviors. The correlations bearing on these relationships are reported in Table 1.

The first correlation indicates a strong positive relationship between how much kindness and how much intelligence are generally conveyed by the same behavior. It should be pointed out, however, that this pattern is somewhat irregular across the six behavior clusters described above. More specifically, behaviors that predominantly convey kindness information tend also to convey intelligence information. In contrast, behaviors that predominantly convey intelligence information do not always convey kindness information. Researchers should exert caution in their stimulus selection to ensure that this asymmetrical relationship between perceived kindness and intelligence does not confound their research design.

The second column of correlations in Table 1 indicates that judgments of a behavior's kindness and intelligence are positively related to the judgments of a behavior's goodness. This is a strong relationship that requires little qualification.

The last column of correlations in Table 1 indicates that judgments of a behavior's normality are positively related to the judgments of a behavior's kindness, intelligence, and goodness. Although significant, these correlations are generally lower than the other correlations in the table. It is possible that they have been attenuated by a few behaviors that were judged to be abnormal, precisely because the implied intelligence or kindness of the statement was considered to be uncharacteristically extreme (cf. the intelligent behaviors assigned to Cluster 3 ).

\section{CONCLUSION}

The behaviors and normative ratings presented here are intended to assist researchers who frequently use behavioral information in their experiments to study the processes underlying impression formation and person memory. We offer an organized stimulus base that will enable many investigators to avoid the tedium of having to create and pretest behavioral items before conducting actual studies. However, we should caution researchers that a ready-made stimulus set can never serve as a substitute for a good research design. The trait implications of a behavior can quickly change as a function of the context in which it is presented. This, in turn, can lead to unintended and potentially confounding effects on the dependent variables of interest. To help circumvent these problems, we strongly encourage researchers to consider counterbalanced designs and stimulus replications when using any of the behaviors presented in this paper for their own experimental work.

\section{REFERENCES}

Fumrman, R. W. (1987, May). The effects of cognitive workload on on-line judgments of social category membership. Paper presented at the meeting of the Midwestern Psychological Association, Chicago.

Lichtenstein, M., \& Srull, T. K. (1987). Processing objectives as a determinant of the relationship between recall and judgment. Journal of Experimental Social Psychology, 23, 93-118.

Srull, T. K., Lichtenstein, M., \& Rothbart, M. (1985). Associative storage and retrieval processes in person memory. Joumal of Experimental Psychology: Learning, Memory, \& Cognition, 11, 316-345.

APPENDIX

Mean Goodness, Kindness, Intelligence, and Normality Ratings for $\mathbf{4 0 0}$ Stimulus Behaviors

\begin{tabular}{|c|c|c|c|c|c|c|c|c|c|c|c|c|c|}
\hline \multirow[b]{3}{*}{ Behavior } & \multicolumn{12}{|c|}{ Ratings } & \multirow[b]{3}{*}{ Cluster } \\
\hline & \multicolumn{3}{|c|}{ Goodness } & \multicolumn{3}{|c|}{ Kindness } & \multicolumn{3}{|c|}{ Intelligence } & \multicolumn{3}{|c|}{ Normality } & \\
\hline & $M$ & $S D$ & Rank & $M$ & $S D$ & Rank & $M$ & $S D$ & Rank & $M$ & $S D$ & Rank & \\
\hline $\begin{array}{l}\text { Offered to help an elderly neighbor paint } \\
\text { his house }\end{array}$ & 9.08 & 1.33 & 1 & 9.38 & 0.86 & 1 & 7.86 & 1.63 & 36.5 & 6.46 & 2.39 & 107.0 & 1 \\
\hline $\begin{array}{l}\text { Gave out toys to the Children's Hospital at } \\
\text { Christmas }\end{array}$ & 8.90 & 1.29 & 2 & 8.97 & 1.24 & 4 & 7.43 & 191 & 25 & 71 & 267 & & 1 \\
\hline $\begin{array}{l}\text { Helped a lost child find her parents in a store } \\
\text { Volunteered his time as a big brother to a }\end{array}$ & 8.85 & 1.33 & 3 & 8.65 & 1.11 & 11 & 7.69 & $\begin{array}{l}1.91 \\
1.76\end{array}$ & $\begin{array}{l}02.5 \\
45.5\end{array}$ & $\begin{array}{l}5.11 \\
6.80\end{array}$ & $\begin{array}{l}2.01 \\
2.01\end{array}$ & $\begin{array}{r}143 \\
83\end{array}$ & 1 \\
\hline fatherless child & 8.72 & 1.93 & 4 & 9.03 & 1.09 & 3 & 7.11 & 2.15 & 86.5 & 5.57 & 2.38 & 152.5 & 1 \\
\hline Provided food and clothing for flood victims & 8.64 & 1.90 & 5.5 & 8.76 & 1.80 & 7 & 7.91 & 1.74 & 34.5 & 6.00 & 2.18 & 125 & 1 \\
\hline Visited a nursing home to cheer up the patients & 8.64 & 1.41 & 5.5 & 8.73 & 1.24 & 8 & 7.17 & 2.01 & 82.5 & 4.69 & 2.42 & 210.5 & 1 \\
\hline Gave food to the needy & 8.62 & 1.73 & 7.5 & 8.60 & 1.71 & 14 & 7.94 & 1.91 & 32.5 & 6.97 & 2.16 & 76 & 1 \\
\hline Won the Nobel Peace Prize & 8.62 & 1.90 & 7.5 & 6.76 & 2.06 & 72 & 9.46 & 1.22 & 2 & 0.80 & 1.53 & 400 & 3 \\
\hline Helped an elderly woman put in storm windows & 8.54 & 1.68 & 10 & 8.84 & 1.04 & 5.5 & 7.77 & 1.82 & 40.5 & 6.54 & 2.09 & 98.5 & 1 \\
\hline Visited a sick friend in the hospital & 8.54 & 1.71 & 10 & 8.30 & 1.08 & 29 & 7.49 & 2.08 & 54.5 & 8.63 & 1.56 & 14.5 & 1 \\
\hline Gave his coat to someone when it was cold & 8.54 & 1.83 & 10 & 8.54 & 1.56 & 17 & 6.17 & 2.19 & 145.5 & 5.83 & 2.28 & 137 & 1 \\
\hline
\end{tabular}


APPENDIX (Continued)

\begin{tabular}{|c|c|c|c|c|c|c|c|c|c|c|c|c|c|}
\hline \multirow[b]{3}{*}{ Behavior } & \multicolumn{12}{|c|}{ Ratings } & \multirow[b]{3}{*}{ Cluster } \\
\hline & \multicolumn{3}{|c|}{ Goodness } & \multicolumn{3}{|c|}{ Kindness } & \multicolumn{3}{|c|}{ Intelligence } & \multicolumn{3}{|c|}{ Normality } & \\
\hline & $M$ & $S D$ & $\overline{\text { Rank }}$ & $M$ & $S D$ & $\overline{\text { Rank }}$ & $M$ & $S D$ & Rank & $M$ & $S D$ & $\overline{\text { Rank }}$ & \\
\hline
\end{tabular}
Gave his balloon to a child who had let hers
Talked to a lonely child at the company
picnic for an hour

Worked on a campaign to release prisoners of war

Comforted a man whose wife had recently died

Lent money to a friend in a financial crisis

Ranked Number 1 in his medical school class

Gave money to charity

Helped an older man carry his luggage to his car

Helped a man in a wheelchair cross a busy intersection

Found an expensive briefcase and tried to locate its owner

Kept the music down for his roommates

Worked all summer to give his parents a trip to Hawaii

Spent time voluntarily tutoring disadvantaged students

Went to the grocery store for his sick wife

Shared his umbrella with someone during the rain

Volunteered several hours a week at a runaway shelter

Went out of his way to help a foreigner find the bus station

Stayed up late helping his child with homework

Doesn't smoke in other people's apartments

Made breakfast for his wife on her birthday

Offered to help a neighbor fix a fence

Picked up a child so that she could see the parade

Turned the lights off in a parked car

Had 10 job offers upon graduating from college

Was valedictorian of his graduating class

Always remembers the birthdays of friends and relatives

Helped a neighbor fix his roof

Volunteered to help renovate a church

Provided books to a nursing home

Gave his seat to someone on the crowded bus

Paid for a friend's dinner

Helped push someone's car out of a snow bank

Participated in an effort to clean up a city park

Gave a beggar a five-dollar bill at

Christmas time

Was accepted into Harvard's Law School

Volunteered to stay late to help a coworker

Rarely misses an appointment

Scored in the 99th percentile on the GRE Exam

Gave someone directions

Helped pick up the papers dropped by another

Successfully managed his own stock market investments

Volunteered his time for a campaign to help save the seals

Smiled and greeted people while walking down the street

Tried to involve a shy friend in social activities

Bought food for everyone in the office

Graduated magna cum laude

Allowed an evicted friend to stay with him temporarily

$\begin{array}{llll}7.67 & 1.95\end{array}$

Stopped to let another car into the line of traffic $7.64 \quad 1.77$
12

$8.49 \quad 1.55 \quad 13$

$\begin{array}{ll}8.46 & 1.88\end{array}$

$8.39 \quad 1.58$

$\begin{array}{ll}8.36 & 1.95\end{array}$

$8.36 \quad 1.98$

$8.33 \quad 1.48$

$8.33 \quad 1.64$

$8.33 \quad 1.60$

$8.33 \quad 1.48$

$8.31 \quad 1.42$

$8.31 \quad 1.92$

$8.28 \quad 1.70$

$8.26 \quad 2.07$

$8.26 \quad 1.62$

$8.18 \quad 1.72$

$8.18 \quad 1.89$

$\begin{array}{ll}8.15 & 1.27\end{array}$

$8.15 \quad 1.71$

$8.13 \quad 1.40$

$8.08 \quad 1.66$

$8.05 \quad 1.52$

$8.03 \quad 1.69$

$8.03 \quad 2.08$

$8.03 \quad 1.94$

$\begin{array}{ll}7.92 & 1.51\end{array}$

$\begin{array}{ll}7.92 & 1.29\end{array}$

$\begin{array}{ll}7.92 & 1.71\end{array}$

$\begin{array}{ll}7.90 & 1.43\end{array}$

$7.90 \quad 2.26$

$7.87 \quad 1.51$

$\begin{array}{ll}7.87 & 1.92\end{array}$

$\begin{array}{ll}7.87 & 1.54\end{array}$

$\begin{array}{ll}7.87 & 1.66\end{array}$

$\begin{array}{ll}7.85 & 2.10\end{array}$

$\begin{array}{ll}7.82 & 1.37\end{array}$

$\begin{array}{ll}7.80 & 1.81\end{array}$

$\begin{array}{ll}7.80 & 2.05\end{array}$

$7.77 \quad 1.63$

$7.77 \quad 1.88$

$7.77 \quad 1.65$

$7.74 \quad 1.87$

$\begin{array}{ll}7.72 & 1.49\end{array}$

$7.69 \quad 1.42$

$7.69 \quad 1.96$

$7.69 \quad 2.40$

\begin{tabular}{ll}
$7.67 \quad 1.95$ \\
\hline
\end{tabular} $\begin{array}{llllll}8.14 & 1.42 & 36.5 & 6.49 & 1.62 & 122\end{array}$

$\begin{array}{lll}6.89 & 1.71 \quad 79\end{array}$

$13 \quad 8.57 \quad 1.36$

$\begin{array}{lll}15 & 7.77 \quad 1.90\end{array}$

40.5

$\begin{array}{lll}14 & 8.38 & 1.50\end{array}$

$\begin{array}{llll}28 & 6.86 & 2.09 & 105\end{array}$

$\begin{array}{lll}15 & 8.40 & 1.54\end{array}$

$\begin{array}{lll}16.5 & 8.49 & 1.46\end{array}$

$\begin{array}{lll}16.5 & 5.89 & 1.54\end{array}$

$\begin{array}{lll}19.5 & 8.54 & 1.24\end{array}$

$\begin{array}{lll}19.5 & 8.49 & 1.26\end{array}$

$\begin{array}{lll}25.5 & 8.00 & 1.75\end{array}$

$\begin{array}{lll}19.5 & 7.09 & 2.06\end{array}$

$\begin{array}{ccc}107.5 & 9.77 & 0.60\end{array}$

$\begin{array}{lll}17 & 6.60 & 1.70\end{array}$

$\begin{array}{lll}19.5 & 7.54 & 1.92\end{array}$

$\begin{array}{lll}19.5 & 8.84 & 1.21\end{array}$

$\begin{array}{lll}5.5 & 7.54 & 1.79\end{array}$

$\begin{array}{lll}19.5 & 8.70 & 1.22\end{array}$

$\begin{array}{lll}9 & 7.63 & 2.14\end{array}$

$\begin{array}{lll}22.5 & 7.65 & 1.64\end{array}$

55

$\begin{array}{ll}7.14 & 1.78\end{array}$

$\begin{array}{lll}22.5 & 9.22 & 0.92\end{array}$

$2 \quad 7.40 \quad 2.25$

$\begin{array}{lll}24 & 8.65 & 1.44\end{array}$

$\begin{array}{lll}11 & 8.14 & 1.61\end{array}$

$\begin{array}{llll}05 & 3.57 & 2.29 & 294\end{array}$

$\begin{array}{llll}31 & 8.43 & 1.67 & 22\end{array}$

$\begin{array}{llll}90 & 7.00 & 2.09 & 73.5\end{array}$

$\begin{array}{llll}1 & 1.57 & 2.09 & 391\end{array}$

$\begin{array}{llll}116 & 7.57 & 1.99 & 43\end{array}$

$\begin{array}{llll}51.5 & 6.57 & 2.00 & 95.5\end{array}$

$\begin{array}{lll}25.5 & 8.43 & 1.34\end{array}$

$\begin{array}{lll}22 & 8.06 & 1.81\end{array}$

$\begin{array}{llll}51.5 & 6.31 & 2.01 & 112.5\end{array}$

$\begin{array}{lll}25.5 & 8.08 & 1.40\end{array}$

$\begin{array}{lll}39.5 & 6.97 & 1.77\end{array}$

$\begin{array}{lll}27.5 & 8.62 & 1.30\end{array}$

13

$\begin{array}{ll}7.11 & 2.03\end{array}$

$\begin{array}{llll}49 & 6.20 & 2.18 & 120\end{array}$

$\begin{array}{llll}84.5 & 7.14 & 1.68 & 63.5\end{array}$

$\begin{array}{lll}27.5 & 8.40 & 1.40\end{array}$

$\begin{array}{lll}29.5 & 8.40 & 1.28\end{array}$

$\begin{array}{lll}29.5 & 7.81 & 1.70\end{array}$

$\begin{array}{lll}31 & 8.22 & 1.60\end{array}$

$\begin{array}{lll}32 & 7.81 & 1.35\end{array}$

$\begin{array}{lll}33 & 8.27 & 1.17\end{array}$

$\begin{array}{lll}35 & 7.62 & 1.55\end{array}$

$\begin{array}{lll}35 & 5.81 & 1.61\end{array}$

$\begin{array}{lll}35 & 5.73 & 1.33\end{array}$

$\begin{array}{lll}25.5 & 6.63 & 1.80\end{array}$

$\begin{array}{llll}25.5 & 8.11 & 1.62\end{array}$

$\begin{array}{lll}48 & 7.43 & 2.21\end{array}$

$\begin{array}{lll}33 & 6.40 & 1.94\end{array}$

$\begin{array}{llll}48 & 6.40 & 1.50 & 131\end{array}$

$\begin{array}{llll}66.5 & 3.26 & 2.68 & 317.5\end{array}$

$\begin{array}{llll}24.5 & 4.49 & 2.04 & 227.5\end{array}$

$\begin{array}{lll}31 & 7.43 & 2.09\end{array}$

$\begin{array}{lll}57.5 & 7.46 & 1.76\end{array}$

$\begin{array}{lll}115 & 8.26 & 1.50\end{array}$

$\begin{array}{lll}130 & 9.11 & 1.02\end{array}$

$\begin{array}{lll}38 & 7.97 & 1.52\end{array}$

$\begin{array}{lll}38 & 8.08 & 1.62\end{array}$

$\begin{array}{lll}38 & 8.54 & 1.12\end{array}$

$\begin{array}{lll}40.5 & 7.78 & 1.73\end{array}$

$\begin{array}{lll}40.5 & 8.14 & 1.53\end{array}$

$\begin{array}{lll}43.5 & 7.86 & 1.53\end{array}$

$\begin{array}{lll}43.5 & 8.43 & 1.46\end{array}$

$\begin{array}{lll}43.5 & 8.27 & 1.30\end{array}$

$\begin{array}{lll}43.5 & 8.40 & 1.28\end{array}$

$\begin{array}{lll}46 & 5.89 & 1.56\end{array}$

$\begin{array}{lll}47 & 8.27 & 1.46\end{array}$

$\begin{array}{lll}48.5 & 6.24 & 1.40\end{array}$

$\begin{array}{lll}43.5 & 7.09 & 1.72\end{array}$

$\begin{array}{lll}39.5 & 7.31 & 1.76\end{array}$

$\begin{array}{lll}17 & 6.83 & 1.50\end{array}$

$\begin{array}{lll}50 & 7.06 & 1.66\end{array}$

$\begin{array}{lll}36.5 & 6.06 & 1.97\end{array}$

$\begin{array}{llll}46 & 5.57 & 1.40\end{array}$

$\begin{array}{llllllll}31 & 7.31 & 1.76 & 70.5 & 5.54 & 1.95 & 157.5\end{array}$

$\begin{array}{lll}48.5 & 5.68 & 1.63\end{array}$

$\begin{array}{lll}51 & 7.38 & 1.36\end{array}$

$\begin{array}{lll}25.5 & 6.74 & 1.79\end{array}$

$\begin{array}{llll}111.5 & 5.37 & 1.93 & 166\end{array}$

$\begin{array}{lll}107.5 & 9.43 & 0.98\end{array}$

$\begin{array}{lll}31 & 7.43 & 1.65\end{array}$

$\begin{array}{llll}3 & 3.06 & 2.27 & 325.5\end{array}$

$\begin{array}{llll}62.5 & 6.06 & 2.11 & 123\end{array}$

$\begin{array}{llll}90 & 6.57 & 2.06 & 95.5\end{array}$

$\begin{array}{rrrr}5 & 1.77 & 1.59 & 386.5\end{array}$

$\begin{array}{lll}134.5 & 9.17 & 1.45\end{array}$

$\begin{array}{lll}64.5 & 7.26 & 1.62\end{array}$

$\begin{array}{rrrr}76.5 & 8.29 & 1.66 & 25\end{array}$

$\begin{array}{lll}51 & 7.62 & 1.59\end{array}$

$\begin{array}{lll}57.5 & 7.26 & 1.76\end{array}$

$\begin{array}{lll}51 & 5.43 & 0.93\end{array}$

176

$8.29 \quad 1.84$

76.57 .06

$\begin{array}{lll}53 & 8.16 & 1.24\end{array}$

$\begin{array}{lll}34.5 & 7.43 & 1.89\end{array}$

19

$\begin{array}{lll}5.54 & 2.43 & 157.5\end{array}$

$\begin{array}{lll}54 & 7.60 & 1.12\end{array}$

$\begin{array}{lllllll}59 & 6.57 & 1.84 & 117.5 & 6.49 & 2.73 & 104.5\end{array}$

$\begin{array}{lll}56 & 7.65 & 1.36\end{array}$

$\begin{array}{lll}56 & 8.16 & 1.38\end{array}$

$\begin{array}{lllllll}55 & 7.46 & 1.50 & 57.5 & 7.31 & 1.35 & 52.5\end{array}$

$\begin{array}{lll}56 & 5.68 & 1.43\end{array}$

$\begin{array}{lllllll}34.5 & 5.49 & 1.60 & 197.5 & 4.23 & 2.35 & 243.5\end{array}$

$\begin{array}{lll}58.5 & 7.65 & 1.89\end{array}$

$\begin{array}{lll}58.5 & 8.11 & 1.26\end{array}$

$\begin{array}{lll}61 & 7.27 & 1.48\end{array}$

55

$\begin{array}{llllll}6.14 & 2.16 & 148.5 & 6.40 & 2.23 & 110.5\end{array}$

$\begin{array}{llllllll}38 & 7.14 & 1.72 & 84.5 & 6.17 & 1.79 & 122\end{array}$

66

$\begin{array}{lllllr}6.06 & 2.01 & 155.5 & 7.20 & 2.10 & 61\end{array}$

1

1

1

1

1

1

1

1

1

1 
APPENDIX (Continued)

\begin{tabular}{|c|c|c|c|c|c|c|c|c|c|c|c|c|c|}
\hline \multirow[b]{3}{*}{ Behavior } & \multicolumn{12}{|c|}{ Ratings } & \multirow[b]{3}{*}{ Cluster } \\
\hline & \multicolumn{3}{|c|}{ Goodness } & \multicolumn{3}{|c|}{ Kindness } & \multicolumn{3}{|c|}{ Intelligence } & \multicolumn{3}{|c|}{ Normality } & \\
\hline & $M$ & $S D$ & Rank & $M$ & $S D$ & Rank & $M$ & $S D$ & Rank & $M$ & $S D$ & Rank & \\
\hline
\end{tabular}

Discovered a cheap way of producing solar energy

$7.64 \quad 1.63$

Put money in the meter for an unknown driver 7.642 .31

Created a new computer language

Told the owner of a store that she gave him

too much change

Gave a stranded motorist a lift to the service station

Invited an unpopular coworker to go to lunch with him

Sang at a prison in order to entertain the inmates

Placed first in a spelling bee

Rescued an injured kitten from a tree

Received a PhD in biology

Offered to show a new student around campus after class

Did all of the repair work on his own car

Fixed a friend's broken typewriter

Stuck to his diet for several weeks

Successfully defended himself in a court case

Plays five different musical instruments

Won a scholarship from the National

Science Foundation

Helped a neighbor move his piano

Can speak four languages

Translated menu items for a foreigner in a restaurant

Took a friend out for a beer to celebrate his promotion

Was voted most likely to succeed by members of his class

Built a solar collector for heating water in his home

Won a science fiction writing award

Received an award for a money-saving job idea

Circulated a petition in support of civil rights

Let a civic group hold weekly meetings in his home

Was in the school's honor society

Spent time one summer teaching his daughter to play baseball

Rode his bike to work every day to help cut down pollution

Complimented coworkers on their clothing and appearance

Brushes his teeth after each meal

Vacationed in Europe with his parents

Went for a picnic in the park

Goes to church on a regular basis

Left food out for stray dogs

Opened the door to cool the room

Gave a lecture on astrophysics to a high school science club

Reads several classic novels every summer

Published an essay in a national magazine

Studied photography in his spare time

Beat the professor in a class argument

Dusted his living room before a party

Designed the blueprint for his home

Can solve the Rubik's cube in 4 minutes

Could name the capital cities of half of the United States

Has written five books

Went for a walk on the first warm day in March

$\begin{array}{ll}7.62 & 2.29\end{array}$

$\begin{array}{ll}7.54 & 2.08\end{array}$

$7.54 \quad 1.90$

$\begin{array}{ll}7.51 \quad 1.70 \\ 7.49 & 2.43\end{array}$

$7.49 \quad 2.43$

$7.49 \quad 2.30$

$\begin{array}{ll}7.44 & 1.76\end{array}$

$\begin{array}{ll}7.44 & 1.70\end{array}$

$\begin{array}{ll}7.41 & 1.83\end{array}$

$\begin{array}{ll}7.41 & 2.09\end{array}$

$7.41 \quad 1.71$

$\begin{array}{ll}7.36 & 2.38\end{array}$

$\begin{array}{ll}7.31 & 1.56\end{array}$

$7.28 \quad 1.81$

$7.23 \quad 1.65$

$7.23 \quad 4.58$

$\begin{array}{ll}7.21 & 2.00\end{array}$

$\begin{array}{ll}7.08 & 1.49\end{array}$

\begin{tabular}{ll}
$7.05 \quad 1.40$ \\
\hline
\end{tabular}

$7.05 \quad 1.67$

$\begin{array}{ll}7.03 & 1.51\end{array}$

$7.03 \quad 1.95$

$6.97 \quad 1.87$

$\begin{array}{ll}6.95 \quad 2.08 \\ 6.95 & 1.56\end{array}$

$\begin{array}{ll}6.95 & 1.56\end{array}$

$6.92 \quad 1.75$

$6.90 \quad 1.96$

$\begin{array}{ll}6.87 & 2.02\end{array}$

$\begin{array}{ll}6.85 & 1.77\end{array}$

$6.80 \quad 1.70$

$6.80 \quad 1.69$

$\begin{array}{ll}6.80 & 1.87\end{array}$

$6.77 \quad 1.81$

$\begin{array}{ll}6.77 & 2.24\end{array}$

$6.72 \quad 1.45$

$6.69 \quad 1.52$

$6.69 \quad 1.91$

$6.67 \quad 1.69$
61

$\begin{array}{lll}61 & 7.68 & 2.12\end{array}$

$\begin{array}{llll}63 & 5.95 & 1.67 & 103\end{array}$

$\begin{array}{ll}7.56 & 1.97\end{array}$

$\begin{array}{lll}64 & 7.89 & 1.81\end{array}$

45

66

$\begin{array}{lll}8.65 & 0.92 \quad 11\end{array}$

$\begin{array}{lll}66 & 7.68 & 1.58\end{array}$

$\begin{array}{lll}66 & 7.51 & 1.95\end{array}$

$\begin{array}{lll}68 & 5.35 & 1.23\end{array}$

$\begin{array}{llll}52 & 5.80 & 1.53 & 172\end{array}$

$\begin{array}{llll}61.5 & 6.23 & 1.63 & 139\end{array}$

$\begin{array}{llll}188.5 & 8.69 & 1.18 & 13\end{array}$

$\begin{array}{llll}69.5 & 8.43 & 1.30 & 22\end{array}$

$\begin{array}{lll}6.71 & 1.93 & 113\end{array}$

$\begin{array}{llll}69.5 & 5.84 & 1.69 & 113\end{array}$

$9.00 \quad 1.19$

$\begin{array}{llll}71.5 & 8.05 & 1.20\end{array}$

$\begin{array}{lll}4.20 & 1.75\end{array}$

$\begin{array}{llllll}71.5 & 5.78 & 1.48 & 118.5 & 8.31 & 1.43\end{array}$

$\begin{array}{lllllll}74.5 & 7.57 & 1.28 & 60 & 7.67 & 1.53\end{array}$

$\begin{array}{llllll}74.5 & 5.86 & 1.57 & 110 & 7.06 & 2.07\end{array}$

$\begin{array}{llllll}74.5 & 5.40 & 1.28 & 180 & 8.91 & 1.15\end{array}$

$\begin{array}{llllll}74.5 & 5.95 & 1.49 & 103 & 7.49 & 1.31\end{array}$

77

$\begin{array}{lll}5.73 & 1.41 & 130\end{array}$

$\begin{array}{llllll}8.77 & 1.09 & 11.5 & 3.26 & 2.64 & 317.5\end{array}$

78

$\begin{array}{lll}8.03 & 1.38 & 42\end{array}$

$6.03 \quad 1.92$

$\begin{array}{llllll}5.78 & 1.44 & 118.5 & 8.91 & 1.04\end{array}$

$\begin{array}{llll}80 & 5.46 & 1.95 & 162.5\end{array}$

$\begin{array}{ll}7.26 & 1.83\end{array}$

$\begin{array}{lll}80 & 7.97 & 1.50\end{array}$

$\begin{array}{lll}43.5 & 7.34 & 1.35\end{array}$

$81.5 \quad 7.19 \quad 1.61$

67

$6.11 \quad 1.51$

$\begin{array}{llll}68 & 4.54 & 2.63 & 222\end{array}$

$\begin{array}{lll}81.5 & 6.78 & 1.95\end{array}$

71

$7.71 \quad 1.76$

150

83

$5.49 \quad 1.12$

$\begin{array}{lll}164.5 & 8.20 & 1.30\end{array}$

84

86

$5.49 \quad 1.12$

$\begin{array}{lll}164.5 & 7.94 & 1.64\end{array}$

$6.08 \quad 1.52$

$\begin{array}{lll}87.5 & 7.40 & 1.50\end{array}$

$\begin{array}{lll}86 & 6.97 & 1.44\end{array}$

$\begin{array}{lll}68.5 & 6.51 & 1.99\end{array}$

$\begin{array}{lllll}86 & 7.68 & 1.45 & 52\end{array}$

$\begin{array}{ll}6.17 & 1.74\end{array}$

$119.5 \quad 4.43$

$\begin{array}{llll}88.5 & 5.54 & 1.22 & 155\end{array}$

$7.91 \quad 1.72$

$\begin{array}{lll}88.5 & 7.51 \quad 1.66\end{array}$

$\begin{array}{lll}90 & 6.70 & 2.25\end{array}$

$\begin{array}{lll}61.5 & 7.31 & 1.83\end{array}$

$\begin{array}{lll}91.5 & 7.38 & 1.14\end{array}$

$\begin{array}{lll}73 & 7.06 & 2.18\end{array}$

$\begin{array}{llll}70.5 & 6.31 & 2.45 & 112.5\end{array}$

$6.90 \quad 1.93$

$\begin{array}{lll}91.5 & 6.05 & 1.60\end{array}$

$\begin{array}{lll}93 & 5.65 & 1.38\end{array}$

$\begin{array}{llll}94.5 & 5.60 & 1.55\end{array}$

$\begin{array}{llll}64.5 & 6.89 & 1.81 & 104\end{array}$

$\begin{array}{lll}94.5 & 6.08 & 1.48\end{array}$

$\begin{array}{lll}96 & 7.81 & 1.70\end{array}$

$\begin{array}{llll}97 & 5.76 & 1.28\end{array}$

$\begin{array}{llll}89.5 & 7.29 & 1.71 & 74\end{array}$

$\begin{array}{llll}99 & 6.65 & 1.27 & 74\end{array}$

$\begin{array}{lllll}99 & 5.19 & 0.81 & 209 & 8.77\end{array}$

$\begin{array}{clllll}99 & 5.19 & 0.81 & 209 & 8.77 & 1.31 \\ 101.5 & 5.38 & 1.01 & 184.5 & 6.31 & 1.28\end{array}$

.28

$\begin{array}{lllllll}101.5 & 5.24 & 1.28 & 204 & 7.69 & 2.07\end{array}$

$\begin{array}{lll}103 & 6.22 & 1.87\end{array}$

$\begin{array}{lll}82.5 & 6.34 & 1.61\end{array}$

$\begin{array}{lll}104.5 & 5.38 & 1.36\end{array}$

$\begin{array}{lll}184.5 & 8.40 & 1.06\end{array}$

02

$\begin{array}{llllllllll}106.5 & 5.11 & 1.00 & 220 & 6.20 & 1.66 & 142 & 5.29 & 2.33 & 171 \\ 106.5 & 5.49 & 1.10 & 164.5 & 8.06 & 1.76 & 28.5 & 2.00 & 2.14 & 378\end{array}$

$\begin{array}{llllllll}84.5 & 8.40 & 1.06 & 16.5 & 3.80 & 2.45 & 278.5 & 4 \\ 70.5 & 6.83 & 2.02 & 107 & 3.71 & 2.16 & 287.5 & 4\end{array}$ $\begin{array}{llllllllll}109 & 5.76 & 1.48 & 124.5 & 7.31 & 1.81 & 70.5 & 8.54 & 1.69 & 18.5\end{array}$ $\begin{array}{llll}18 & 5.11 & 2.26 & 179\end{array}$

$\begin{array}{llll}47 & 5.51 & 2.31 & 160\end{array}$

$\begin{array}{llll}94.5 & 4.89 & 2.35 & 193.5\end{array}$

$\begin{array}{llll}8.5 & 4.11 & 2.81 & 250.5\end{array}$

$\begin{array}{llll}54.5 & 2.54 & 2.32 & 357\end{array}$

$\begin{array}{llll}159 & 6.26 & 2.20 & 115.5\end{array}$

$\begin{array}{llll}8.5 & 1.91 & 1.74 & 383\end{array}$

$\begin{array}{llll}21 & 2.51 & 2.27 & 359.5\end{array}$

$\begin{array}{llll}66.5 & 4.83 & 2.49 & 197.5\end{array}$

$\begin{array}{llll}145.5 & 3.83 & 2.55 & 277\end{array}$

$\begin{array}{llll}34.5 & 6.74 & 2.01 & 86.5\end{array}$

$94.5 \quad 3.26 \quad 2.20 \quad 317.5$

$\begin{array}{lll}7.03 & 2.27 & 70\end{array}$

74
69

$\begin{array}{lll}6.26 & 2.89 & 115.5\end{array}$

$\begin{array}{lll}4.37 & 2.35 & 237.5\end{array}$

$\begin{array}{lll}6.77 & 2.13 & 85\end{array}$

3

1

1

1

1

1

4

3 1

1

1

4

1

6

4

1

4

$\begin{array}{lllll}43 & 4.43 & 2.37 & 232 & 4 \\ 11.5 & 3.23 & 2.66 & 320.5 & 4\end{array}$

$\begin{array}{lllll}136 & 5.57 & 2.38 & 152.5 & 4\end{array}$

$\begin{array}{lllll}45.5 & 3.77 & 2.34 & 281.5 & 4\end{array}$

$\begin{array}{llllll}35 & 8.83 & 1.51 & 11 & 6\end{array}$

4

4
3
6 
APPENDIX (Continued)

\begin{tabular}{|c|c|c|c|c|c|c|c|c|c|c|c|c|c|}
\hline & & & & & & & & & & \multirow{2}{*}{\multicolumn{3}{|c|}{ Normality }} & \\
\hline & \multicolumn{3}{|c|}{ Goodness } & \multicolumn{3}{|c|}{ Kindness } & \multicolumn{3}{|c|}{ Intelligence } & & & & \multirow[b]{2}{*}{ Cluster } \\
\hline Behavior & $M$ & $S D$ & Rank & $M$ & $S D$ & Rank & $M$ & $S D$ & Rank & $M$ & $S D$ & Rank & \\
\hline
\end{tabular}

Left town on short notice because of his father's death

Edited and published an anthology of literary criticism

Put gas in the car

Stayed late in the chemistry lab to try his own experiments

Hit the ball at a softball game

Can multiply three-digit numbers in his head

Managed the local campaign for a presidential candidate

Laughed at a friend's joke even though it wasn't funny

Planted flowers around his home

Attends many drama performances

Set the curve on the history exam

Developed a complex filing system for his company

Knew which wild plants are edible or poisonous

Went to $\mathrm{Ft}$. Lauderdale for a wintertime vacation

Won a chess tournament

Gave up smoking at the age of 14

Was consulted by a local company for his ideas on a project

Received an honorable mention at a science fair

Swam 20 laps during lunch hour

Told a joke at a party

Runs a mile every day

Repaired a digital clock

Went for a summer vacation in Colorado

Tried out for the lead in a play

Sailed his boat across Lake Michigan

Went on a canoe trip in New England

Knew the voting record of the senators from his home state

Went to the mountains for a vacation

Writes for the school newspaper

Represented his school in a debate tournament

Made a glass of tea after work

Worked in a state park for a summer

Was invited to present a paper to a group of faculty members

Went scuba diving off the coast of Florida

Tied his shoelaces

Worked in a state park for a summer

Went to a high school reunion

Enjoyed star-gazing on clear summer nights

Fixed a leaky faucet

Emptied the trash before leaving for class

Writes poetry in his spare time

Converts measurements to the metric system in his head

Made a presentation on efficient ways of planning activities

Went to the grocery store with his kids

Read War and Peace over spring vacation

Got a short haircut at the beginning of the summer

Often could solve murder mystery novels

Watched the news for tomorrow's weather forecast

Replaced the kitchen light bulb

$\begin{array}{rllllllllllll}6.64 & 2.57 & 109 & 6.97 & 2.02 & 68.5 & 7.03 & 2.14 & 97 & 8.00 & 2.84 & 32 & 6 \\ 6.64 & 2.49 & 109 & 5.51 & 1.19 & 160 & 8.03 & 1.74 & 30 & 2.40 & 2.78 & 362 & 3 \\ 6.60 & 1.93 & 111 & 5.95 & 1.60 & 103 & 6.97 & 1.81 & 99.5 & 9.40 & 1.04 & 1 & 4 \\ 6.59 & 1.90 & 112 & 5.78 & 1.40 & 118.5 & 7.51 & 1.77 & 53 & 4.00 & 2.29 & 263.5 & 4 \\ 6.56 & 1.89 & 113.5 & 5.57 & 1.32 & 150 & 5.83 & 1.27 & 170.5 & 8.09 & 1.81 & 30 & 6\end{array}$

$\begin{array}{rrlllllllllll}6.56 & 1.60 & 113.5 & 5.68 & 1.49 & 134.5 & 7.60 & 1.63 & 50 & 3.89 & 2.97 & 274 & 4\end{array}$

$\begin{array}{lllllllllllll}6.51 & 1.54 & 118.5 & 7.43 & 1.44 & 63 & 5.49 & 1.56 & 197.5 & 7.86 & 1.94 & 34.5 & 1\end{array}$

$\begin{array}{llllllllllllll}6.51 & 1.45 & 118.5 & 6.11 & 1.37 & 85.5 & 5.69 & 1.81 & 180.5 & 7.43 & 2.12 & 49 & 6\end{array}$

$\begin{array}{llllllllllllll}6.51 & 1.65 & 118.5 & 5.54 & 1.30 & 155 & 6.37 & 1.22 & 133.5 & 5.26 & 1.98 & 172 & 4\end{array}$

$\begin{array}{lllllllllllll}6.51 & 2.09 & 118.5 & 5.05 & 1.81 & 231.5 & 7.86 & 2.17 & 36.5 & 5.09 & 2.83 & 181.5 & 4\end{array}$

$\begin{array}{llllllllllllll}6.51 & 1.95 & 118.5 & 5.76 & 1.23 & 124.5 & 8.40 & 1.65 & 16.5 & 4.03 & 2.32 & 258.5 & 4\end{array}$

$\begin{array}{lllllllllllll}6.51 & 1.25 & 118.5 & 5.95 & 1.33 & 103 & 7.20 & 1.30 & 80 & 3.34 & 2.10 & 312 & 4\end{array}$

$\begin{array}{lllllllllllll}6.49 & 1.70 & 123 & 5.86 & 1.60 & 110 & 6.14 & 1.72 & 148.5 & 7.54 & 2.34 & 44 & 6\end{array}$

$\begin{array}{llllllllllllll}6.49 & 1.57 & 123 & 5.14 & 0.89 & 216.5 & 8.14 & 1.40 & 24.5 & 3.94 & 2.24 & 269 & 4\end{array}$

$\begin{array}{lllllllllllll}6.49 & 2.50 & 123 & 6.05 & 1.62 & 89.5 & 6.97 & 2.45 & 99.5 & 3.31 & 3.08 & 315 & 4\end{array}$

$\begin{array}{lllllllllllll}6.44 & 1.47 & 125 & 5.46 & 1.10 & 170.5 & 8.17 & 1.07 & 22.5 & 5.03 & 2.56 & 185 & 4\end{array}$

$\begin{array}{lllllllllllll}6.41 & 1.46 & 126.5 & 5.57 & 1.26 & 150 & 7.66 & 1.61 & 48 & 5.57 & 2.13 & 152.5 & 4\end{array}$

$\begin{array}{lllllllllllll}6.41 & 1.43 & 126.5 & 5.57 & 1.19 & 150 & 6.23 & 1.75 & 139 & 4.03 & 2.33 & 258.5 & 6\end{array}$

$\begin{array}{llllllllllllll}6.36 & 1.58 & 129 & 5.65 & 1.38 & 139 & 5.31 & 0.72 & 208 & 8.23 & 1.65 & 27 & 6\end{array}$

$\begin{array}{llllllllllllll}6.36 & 1.35 & 129 & 5.54 & 1.10 & 155 & 6.46 & 1.92 & 126.5 & 5.31 & 2.40 & 170 & 4\end{array}$

$\begin{array}{llllllllllllll}6.36 & 2.02 & 129 & 5.43 & 0.69 & 176 & 7.09 & 1.04 & 90 & 3.74 & 2.28 & 285 & 4\end{array}$

$\begin{array}{llllllllllllll}6.31 & 1.49 & 132 & 5.86 & 1.44 & 110 & 5.94 & 1.59 & 165.5 & 6.23 & 2.49 & 117.5 & 6\end{array}$

$\begin{array}{llllllllllllll}6.31 & 1.49 & 132 & 5.51 & 1.54 & 160 & 6.29 & 1.41 & 137 & 6.20 & 2.00 & 120 & 4\end{array}$

$\begin{array}{llllllllllllll}6.31 & 1.40 & 132 & 5.84 & 1.30 & 113 & 6.46 & 1.50 & 126.5 & 5.49 & 2.94 & 161 & 4\end{array}$

$\begin{array}{lllllllllllll}6.26 & 2.21 & 134.5 & 5.65 & 1.46 & 139 & 5.83 & 1.51 & 170.5 & 3.40 & 2.50 & 306 & 4\end{array}$

$\begin{array}{llllllllllllll}6.26 & 1.65 & 134.5 & 5.46 & 0.93 & 170.5 & 6.80 & 1.45 & 109.5 & 2.89 & 2.21 & 338.5 & 4\end{array}$

$\begin{array}{llllllllllllll}6.23 & 1.80 & 137 & 5.68 & 1.53 & 134.5 & 5.51 & 1.81 & 192.5 & 7.03 & 2.22 & 70 & 6\end{array}$

$\begin{array}{lllllllllllll}6.23 & 1.33 & 137 & 5.84 & 1.24 & 113 & 6.97 & 1.82 & 99.5 & 5.71 & 2.15 & 143 & 4\end{array}$

$\begin{array}{llllllllllllll}6.23 & 1.22 & 137 & 6.11 & 1.60 & 85.5 & 6.80 & 1.68 & 109.5 & 5.34 & 2.24 & 168 & 4\end{array}$

$\begin{array}{lllllllllllll}6.21 & 1.78 & 140 & 5.43 & 1.24 & 176 & 5.37 & 0.84 & 202.5 & 6.54 & 2.41 & 98.5 & 6\end{array}$

$\begin{array}{lllllllllllll}6.21 & 1.36 & 140 & 5.73 & 1.52 & 130 & 6.46 & 1.70 & 126.5 & 5.06 & 1.98 & 183.5 & 4\end{array}$

$\begin{array}{lllllllllllll}6.21 & 1.59 & 140 & 6.03 & 1.55 & 91.5 & 7.43 & 1.69 & 62.5 & 4.89 & 2.08 & 193.5 & 4\end{array}$

$\begin{array}{llllllllllllll}6.18 & 1.36 & 142 & 5.76 & 1.55 & 124.5 & 6.09 & 1.60 & 152.5 & 4.97 & 2.11 & 189.5 & 4\end{array}$

$\begin{array}{lllllllllllll}6.15 & 2.01 & 143.5 & 5.35 & 1.48 & 188.5 & 5.66 & 1.35 & 184 & 9.20 & 1.83 & 2 & 6\end{array}$

$\begin{array}{llllllllllllll}6.15 & 1.25 & 143.5 & 5.95 & 1.27 & 103 & 6.17 & 1.93 & 145.5 & 5.00 & 2.06 & 187 & 1\end{array}$

$\begin{array}{lllllllllllll}6.13 & 1.63 & 146 & 6.24 & 1.62 & 79.5 & 5.37 & 0.91 & 202.5 & 7.23 & 1.57 & 58.5 & 6\end{array}$

$\begin{array}{llllllllllllll}6.13 & 1.45 & 146 & 6.19 & 1.60 & 84 & 6.40 & 1.61 & 131 & 6.97 & 2.23 & 76 & 6\end{array}$

$\begin{array}{rlllllllllllll}6.13 & 1.44 & 146 & 6.24 & 1.46 & 79.5 & 6.83 & 2.05 & 107 & 6.71 & 1.92 & 88.5 & 4\end{array}$

$\begin{array}{llllllllllllll}6.10 & 1.68 & 148.5 & 6.81 & 1.70 & 70 & 6.03 & 1.40 & 159 & 5.34 & 2.58 & 168 & 6\end{array}$

$\begin{array}{lllllllllllll}6.10 & 1.47 & 148.5 & 5.62 & 1.42 & 143.5 & 7.46 & 1.52 & 57 & 4.37 & 1.90 & 237.5 & 4\end{array}$

$\begin{array}{lllllllllllll}6.08 & 1.35 & 150.5 & 5.35 & 1.09 & 188.5 & 7.83 & 1.44 & 38.5 & 4.74 & 2.49 & 206 & 4\end{array}$

$\begin{array}{lllllllllllll}6.08 & 1.56 & 150.5 & 6.03 & 1.62 & 91.5 & 6.94 & 1.85 & 102.5 & 4.63 & 2.02 & 216 & 4\end{array}$

$\begin{array}{rlllllllllllll}6.05 & 1.10 & 152.5 & 6.57 & 1.57 & 75 & 5.49 & 1.34 & 197.5 & 7.06 & 2.03 & 66 & 1\end{array}$

$\begin{array}{lllllllllllll}6.05 & 2.10 & 152.5 & 5.40 & 1.36 & 180 & 6.20 & 2.31 & 142 & 2.94 & 3.07 & 335 & 4\end{array}$

$\begin{array}{lllllllllllll}6.00 & 1.62 & 154.5 & 5.60 & 1.30 & 146.5 & 6.09 & 1.46 & 152.5 & 7.66 & 2.09 & 40 & 6\end{array}$ $\begin{array}{lllllllllllll}6.00 & 1.41 & 154.5 & 5.76 & 1.52 & 124.5 & 6.94 & 1.35 & 102.5 & 5.74 & 2.20 & 140.5 & 4\end{array}$

$\begin{array}{lllllllllllll}5.97 & 1.33 & 157.5 & 5.76 & 1.59 & 124.5 & 6.23 & 1.37 & 139 & 8.57 & 2.45 & 17 & 4\end{array}$

$\begin{array}{lllllllllllll}5.97 & 1.51 & 157.5 & 5.95 & 1.51 & 103 & 5.66 & 1.14 & 184 & 8.54 & 1.60 & 18.5 & 6\end{array}$

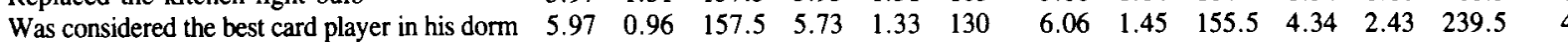

Programmed a computer simulation of the Super Bowl

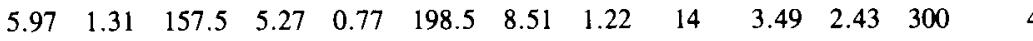


APPENDIX (Continued)

\begin{tabular}{|c|c|c|c|c|c|c|c|c|c|c|c|c|c|}
\hline & \multirow{2}{*}{\multicolumn{3}{|c|}{ Goodness }} & & & & & & & & & & \\
\hline \multirow[b]{2}{*}{ Behavior } & & & & \multicolumn{3}{|c|}{ Kindness } & \multicolumn{3}{|c|}{ Intelligence } & \multicolumn{3}{|c|}{ Normality } & \multirow[b]{2}{*}{ Cluster } \\
\hline & $M$ & $S D$ & Rank & $M$ & $S D$ & Rank & $M$ & $S D$ & Rank & $M$ & $S D$ & Rank & \\
\hline
\end{tabular}

Explained national budgetary policy to a colleague

Usually watches the educational television station

Visited the doctor

Travels to other cities as often as possible

Enjoys going to the movies on the weekend

Walked down the sidewalk in campustown

Visited the library several times a week

Oiled a squeaky door

Plays golf every week to relax

Drove for hours to hear a talk on the aerospace program

Turned out the lights before going to bed

Took the stairs rather than the elevator

Planted vegetables in a garden behind his house

Did crossword puzzles in his spare time

Took a nap one afternoon

Attended a class on recent developments in nuclear physics

Tried on a new shirt

Scratched his back

Bought a new car

Built a bookcase in his apartment

Chopped wood for the fireplace

Entered a lottery for tickets to the World Series

Joined two men in a frisbee game in the park

Had a suit altered by the tailor

Found a dollar bill outside his office door

Bought a new album on Monday

Mowed the lawn on a hot day

Saw a funny movie several times

Cleaned out the top drawer of his desk

Often takes two showers a day

Went to an out-of-town convention by train

Laughed at an old Woody Allen movie

Always wins at Trivial Pursuit

Stacked his records in a closet to save room

Wore a button with the name of his favored political candidate

Sang Top-40 songs in the shower

Sharpened his pencil

Caught three trout on a weekend fishing trip

Bought vegetables at a farmer's market

Finished his girlfriend's dessert at a restauran

Watched a thunderstorm from his front porch

Played poker once a month with several friends

Ate an apple every day

Reads The New York Times and The Wall

Street Journal daily

Mailed a letter at the post office

Hung his poster on the wall

Went swimming at a friend's house

Went ice skating in the park

Polished the chrome on his bicycle

Subscribes to several technical journals

Parked his car at a parking meter

Took his clothes to a nearby laundromat

Washed his car

Replaced the ribbon on his typewriter

Uses literary allusions during conversations at parties

Took off his glasses

Ordered spicy food in a Chinese restaurant

Watched football on television

$\begin{array}{lllllllllllll}5.95 & 1.08 & 160.5 & 6.40 & 1.19 & 76 & 8.17 & 1.20 & 22.5 & 4.17 & 2.33 & 247.5 & 4 \\ & & & & & & & & & & & & \\ 5.95 & 1.72 & 160.5 & 5.38 & 1.04 & 184.5 & 6.49 & 1.15 & 122 & 3.77 & 2.14 & 281.5 & 4 \\ 5.92 & 1.61 & 163 & 5.08 & 0.92 & 225.5 & 7.17 & 1.79 & 82.5 & 8.69 & 1.47 & 13 & 6 \\ 5.92 & 1.35 & 163 & 5.30 & 0.94 & 194.5 & 6.69 & 1.98 & 114 & 5.20 & 2.18 & 176 & 6 \\ 5.92 & 1.38 & 163 & 5.51 & 0.99 & 160 & 6.00 & 1.63 & 161.5 & 8.43 & 1.56 & 22 & 6 \\ 5.90 & 1.83 & 165.5 & 5.32 & 1.16 & 192 & 5.51 & 1.38 & 192.5 & 9.09 & 1.70 & 3 & 6 \\ 5.90 & 1.65 & 165.5 & 5.46 & 1.04 & 170.5 & 7.29 & 1.47 & 74 & 5.71 & 2.57 & 143 & 4 \\ 5.87 & 0.95 & 167 & 6.32 & 1.62 & 77 & 5.63 & 1.48 & 186 & 7.31 & 2.07 & 52.5 & 6 \\ 5.85 & 1.18 & 168.5 & 5.35 & 1.21 & 188.5 & 6.51 & 1.63 & 119.5 & 7.46 & 2.72 & 48 & 6\end{array}$

$\begin{array}{llllllllllll}5.85 & 1.69 & 168.5 & 6.00 & 1.63 & 94 & 6.46 & 2.01 & 126.5 & 4.00 & 2.24 & 263.5\end{array}$

$\begin{array}{llllllllllllll}5.82 & 1.34 & 170 & 5.97 & 1.64 & 97.5 & 5.69 & 1.55 & 180.5 & 9.00 & 2.52 & 5 & 6\end{array}$

$\begin{array}{lllllllllllll}5.82 & 1.32 & 170.5 & 6.00 & 1.41 & 94 & 6.11 & 1.43 & 150.5 & 5.89 & 2.17 & 135 & 4\end{array}$

$\begin{array}{lllllllllllll}5.80 & 1.08 & 172.5 & 5.73 & 1.35 & 130 & 5.97 & 1.64 & 163.5 & 7.89 & 1.76 & 33 & 6\end{array}$

$\begin{array}{llllllllllllll}5.80 & 1.44 & 172.5 & 5.16 & 1.04 & 214 & 6.03 & 1.44 & 159 & 5.97 & 2.01 & 128 & 4\end{array}$

$\begin{array}{llllllllllllll}5.77 & 1.48 & 174.5 & 5.27 & 1.28 & 198.5 & 5.60 & 1.04 & 187.5 & 8.26 & 1.69 & 26 & 6\end{array}$

$\begin{array}{llllllllllllll}5.77 & 1.61 & 174.5 & 5.46 & 0.99 & 170.5 & 7.83 & 1.82 & 38.5 & 3.89 & 2.68 & 274 & 4\end{array}$

$\begin{array}{lllllllllllll}5.74 & 1.67 & 176.5 & 5.27 & 0.93 & 198.5 & 5.46 & 0.95 & 200 & 8.89 & 1.78 & 7.5 & 6\end{array}$

$\begin{array}{lllllllllllll}5.74 & 1.71 & 176.5 & 5.46 & 1.30 & 170.5 & 5.14 & 0.60 & 218 & 8.86 & 1.68 & 9.5 & 6\end{array}$

$\begin{array}{lllllllllllll}5.72 & 1.05 & 179 & 5.27 & 1.07 & 198.5 & 6.46 & 1.84 & 126.5 & 7.29 & 2.05 & 54 & 6\end{array}$

$\begin{array}{llllllllllllll}5.72 & 1.19 & 179 & 5.24 & 0.76 & 204 & 6.74 & 1.56 & 111.5 & 5.94 & 2.14 & 131.5 & 4\end{array}$

$\begin{array}{lllllllllllll}5.72 & 1.00 & 179 & 5.97 & 1.32 & 97.5 & 6.00 & 1.85 & 161.5 & 5.80 & 2.10 & 138.5 & 6\end{array}$

$\begin{array}{llllllllllllll}5.69 & 1.70 & 181 & 5.19 & 0.57 & 209.5 & 6.20 & 1.41 & 142 & 7.03 & 2.37 & 70 & 4\end{array}$

$\begin{array}{lllllllllllll}5.67 & 1.11 & 182.5 & 5.78 & 1.20 & 118.5 & 5.34 & 1.08 & 205 & 5.69 & 1.97 & 145.5 & 6\end{array}$

$\begin{array}{lllllllllllll}5.67 & 1.48 & 182.5 & 5.00 & 0.47 & 237 & 5.69 & 1.23 & 180.5 & 7.80 & 2.27 & 36 & 6\end{array}$

$\begin{array}{lllllllllllll}5.64 & 1.11 & 185 & 5.38 & 1.04 & 184.5 & 5.00 & 1.77 & 228 & 4.20 & 2.75 & 245.5 & 5\end{array}$

$\begin{array}{lllllllllllll}5.64 & 1.31 & 185 & 5.11 & 0.74 & 220 & 5.14 & 0.49 & 218 & 7.03 & 2.92 & 70 & 6\end{array}$

$\begin{array}{rrrrrrrrrrrrr}5.64 & 1.74 & 185 & 5.97 & 1.30 & 97.5 & 5.11 & 1.51 & 221.5 & 7.00 & 2.10 & 73.5 & 6\end{array}$

$\begin{array}{llllllllllllll}5.62 & 0.99 & 187.5 & 5.51 & 1.19 & 160 & 5.91 & 1.34 & 167.5 & 7.49 & 1.82 & 47 & 6\end{array}$

$\begin{array}{lllllllllllll}5.62 & 1.04 & 187.5 & 5.57 & 1.17 & 150 & 5.74 & 1.15 & 175.5 & 7.20 & 2.59 & 61 & 6\end{array}$

$\begin{array}{llllllllllllll}5.59 & 1.37 & 189 & 5.49 & 1.24 & 164.5 & 5.51 & 1.76 & 192.5 & 6.40 & 2.12 & 110.5 & 6\end{array}$

$\begin{array}{llllllllllllll}5.56 & 0.94 & 191 & 5.46 & 1.10 & 170.5 & 5.77 & 1.24 & 173.5 & 4.09 & 2.29 & 253 & 5\end{array}$

$\begin{array}{llllllllllllll}5.56 & 1.62 & 191 & 5.78 & 1.27 & 118.5 & 5.29 & 1.15 & 210.5 & 6.57 & 2.67 & 95.5 & 6\end{array}$

$\begin{array}{llllllllllllll}5.56 & 1.52 & 191 & 5.08 & 1.38 & 225.5 & 7.71 & 1.36 & 43 & 4.00 & 2.34 & 263 & 4\end{array}$

$\begin{array}{lllllllllllll}5.54 & 0.88 & 193.5 & 6.24 & 1.57 & 79.5 & 6.17 & 1.60 & 145.5 & 7.37 & 2.18 & 50 & 6\end{array}$

$\begin{array}{lllllllllllll}5.54 & 1.17 & 193.5 & 5.62 & 1.48 & 143.5 & 5.77 & 1.56 & 173.5 & 6.63 & 1.97 & 91 & 6\end{array}$

$\begin{array}{lllllllllllll}5.51 & 1.32 & 195 & 5.32 & 1.45 & 192 & 4.86 & 2.03 & 233 & 6.60 & 2.76 & 92.5 & 6\end{array}$

$\begin{array}{lllllllllllll}5.49 & 1.32 & 199.5 & 5.11 & 0.66 & 220 & 5.06 & 1.55 & 225.5 & 9.06 & 1.24 & 4 & 6\end{array}$

$\begin{array}{lllllllllllll}5.49 & 1.32 & 199.5 & 5.08 & 1.04 & 225.5 & 5.20 & 1.73 & 213.5 & 7.26 & 1.84 & 56 & 6\end{array}$

$\begin{array}{llllllllllllll}5.49 & 1.17 & 199.5 & 5.65 & 0.92 & 139 & 6.06 & 1.78 & 155.5 & 6.86 & 2.24 & 81 & 6\end{array}$

$\begin{array}{llllllllllllll}5.49 & 1.43 & 199.5 & 5.00 & 1.18 & 237 & 4.86 & 1.17 & 233 & 6.80 & 2.27 & 83 & 6\end{array}$

$\begin{array}{llllllllllllll}5.49 & 1.00 & 199.5 & 5.40 & 1.12 & 180 & 5.11 & 1.05 & 221.5 & 6.51 & 2.28 & 101 & 6\end{array}$

$\begin{array}{lllllllllllll}5.49 & 0.94 & 199.5 & 5.78 & 1.48 & 118.5 & 5.20 & 1.05 & 213.5 & 6.49 & 2.36 & 104.5 & 6\end{array}$

$\begin{array}{lllllllllllll}5.49 & 0.89 & 199.5 & 5.24 & 0.80 & 204 & 5.94 & 1.81 & 165.5 & 5.57 & 3.01 & 152.5 & 4\end{array}$

$\begin{array}{llllllllllllll}5.49 & 0.94 & 199.5 & 5.17 & 0.96 & 212 & 7.46 & 1.46 & 57.5 & 5.54 & 2.32 & 157.5 & 4\end{array}$

$\begin{array}{lllllllllllll}5.44 & 1.12 & 205 & 5.57 & 1.34 & 150 & 5.49 & 1.60 & 197.5 & 8.89 & 1.76 & 7.5 & 6\end{array}$

$\begin{array}{llllllllllllll}5.44 & 2.01 & 205 & 5.19 & 1.05 & 209.5 & 5.20 & 1.05 & 213.5 & 7.86 & 2.93 & 34.5 & 6\end{array}$

$\begin{array}{llllllllllllll}5.44 & 0.97 & 205 & 5.54 & 1.41 & 155 & 5.60 & 1.50 & 187.5 & 6.97 & 2.09 & 76 & 6\end{array}$

$\begin{array}{lllllllllllll}5.41 & 1.16 & 208 & 5.62 & 1.30 & 143.5 & 5.31 & 1.34 & 208 & 7.26 & 2.34 & 56 & 6\end{array}$

$\begin{array}{lllllllllllll}5.41 & 0.60 & 208 & 5.62 & 1.36 & 143.5 & 5.51 & 1.07 & 192.5 & 5.97 & 2.68 & 128 & 6\end{array}$

$\begin{array}{lllllllllllll}5.41 & 1.04 & 208 & 5.08 & 0.92 & 225.5 & 7.20 & 1.23 & 80 & 4.57 & 2.55 & 219 & 4\end{array}$

$\begin{array}{llllllllllllll}5.39 & 1.09 & 210 & 5.95 & 1.65 & 103 & 5.97 & 1.51 & 163.5 & 8.63 & 1.90 & 14.5 & 6\end{array}$

$\begin{array}{lllllllllllll}5.36 & 0.96 & 211 & 5.40 & 1.40 & 180 & 5.74 & 1.72 & 175.5 & 7.34 & 1.63 & 51 & 6\end{array}$

$\begin{array}{lllllllllllll}5.33 & 0.62 & 213 & 5.24 & 0.68 & 204 & 5.51 & 1.95 & 192.5 & 8.86 & 1.61 & 9.5 & 6\end{array}$

$\begin{array}{lllllllllllll}5.33 & 0.81 & 213 & 5.65 & 1.32 & 139 & 5.91 & 1.65 & 167.5 & 7.51 & 2.21 & 45.5 & 6\end{array}$

$\begin{array}{lllllllllllll}5.33 & 1.53 & 213 & 5.05 & 0.70 & 231.5 & 6.37 & 1.68 & 133.5 & 4.71 & 2.23 & 208.5 & 4\end{array}$

$\begin{array}{llllllllllllll}5.28 & 1.23 & 215 & 5.14 & 0.42 & 216.5 & 5.09 & 0.74 & 224 & 8.71 & 1.69 & 12 & 6\end{array}$

$\begin{array}{llllllllllllll}5.26 & 1.33 & 216 & 5.30 & 1.18 & 194.5 & 4.83 & 1.51 & 236 & 4.83 & 2.89 & 197.5 & 5\end{array}$

$\begin{array}{lllllllllllll}5.23 & 0.63 & 219 & 5.27 & 1.02 & 198.5 & 5.17 & 1.15 & 216 & 8.60 & 1.58 & 16 & 6\end{array}$ 
APPENDIX (Continued)

\begin{tabular}{|c|c|c|c|c|c|c|c|c|c|c|c|c|c|}
\hline \multirow[b]{3}{*}{ Behavior } & \multicolumn{12}{|c|}{ Ratings } & \multirow[b]{3}{*}{ Clust } \\
\hline & \multicolumn{3}{|c|}{ Goodness } & \multicolumn{3}{|c|}{ Kindness } & \multicolumn{3}{|c|}{ Intelligence } & \multicolumn{3}{|c|}{ Normality } & \\
\hline & $M$ & $S D$ & Rank & $M$ & $S D$ & Rank & $M$ & $S D$ & Rank & $M$ & $S D$ & Rank & \\
\hline Ate a cheeseburger for lunch & 5.23 & 1.53 & 219 & 5.11 & 0.99 & 220 & 4.97 & 0.89 & 229 & 8.11 & 2.42 & 28.5 & \\
\hline Bought popcorn at a basketball game & 5.23 & 0.90 & 219 & 5.54 & 1.14 & 155 & 5.29 & 1.67 & 210.5 & 7.77 & 2.39 & 37 & \\
\hline Bought a remote control for his television set & 5.23 & 0.71 & 219 & 5.16 & 0.83 & 214 & 5.14 & 1.12 & 218 & 7.14 & 1.80 & 63.5 & \\
\hline Watched an old western on the late show & 5.23 & 1.97 & 219 & 5.03 & 0.44 & 235 & 5.11 & 1.02 & 221.5 & 6.89 & 1.80 & 79 & \\
\hline \multicolumn{14}{|l|}{ Referred to common medicines by their } \\
\hline chemical names & 5.18 & 1.62 & 222 & 5.19 & 1.00 & 209.5 & 7.29 & 2.11 & 74 & 3.26 & 1.95 & 317.5 & \\
\hline Took his boots off after a hiking trip & 5.16 & 0.37 & 223 & 5.08 & 1.59 & 225.5 & 5.51 & 1.34 & 192.5 & 8.06 & 2.58 & 31 & \\
\hline Took the bus home from work & 5.15 & 0.71 & 224 & 5.27 & 0.80 & 198.5 & 5.69 & 1.81 & 180.5 & 8.43 & 1.63 & 22 & \\
\hline Stopped at the cookie store on his way home & 5.10 & 0.82 & 225.5 & 5.46 & 1.28 & 170.5 & 4.83 & 1.47 & 236 & 7.20 & 2. 10 & 61 & \\
\hline Got up regularly at $6: 00 \mathrm{a} . \mathrm{m}$ & 5.10 & 1.07 & 225.5 & 5.22 & 0.89 & 207 & 5.34 & 1.3 & 205 & 6.20 & 2.77 & 120 & \\
\hline Called a TV station for weather information & 5.08 & 1.35 & 227 & 4.97 & 0.44 & 241 & 5.31 & 2. & 20 & 5.00 & 2.89 & 187 & \\
\hline Bought a coffee mug with his initials on it & 5.05 & 1.40 & 228 & 5.24 & 0.72 & 204 & 4.86 & 1.14 & 233 & 7.60 & 1.75 & 41.5 & \\
\hline Owned three pairs of sunglasses & 5.03 & 0.96 & 229.5 & 4.97 & 0.37 & 241 & 5.06 & 1.08 & & 6.23 & 2.49 & & \\
\hline for fun & 5.03 & 1.86 & 229.5 & 5.11 & 0.88 & 220 & 7.06 & 2.04 & & 2.66 & 2.50 & & \\
\hline Rides a motorcycle to work & 4.97 & 1.68 & 231 & 4.84 & 0.76 & 260.5 & 5.11 & 2.00 & 221.5 & 5.43 & 2.31 & 164 & \\
\hline \multicolumn{14}{|l|}{ Prefers to watch I Love Lucy rather than } \\
\hline the news & 4.92 & 1.87 & 232 & 5.08 & 1.16 & 225.5 & 4.37 & 1.22 & 247 & 6.49 & 2.55 & 104.5 & \\
\hline Went to the zoo but left early & 4.90 & 1.10 & 233 & 4.92 & 0.68 & 252 & 4.83 & 0.92 & 236 & 6.57 & 2.05 & 95.5 & \\
\hline Watched two soap operas every weekday & & & & & & & & & & & & & \\
\hline & 4.87 & 1.54 & 234.5 & 5.32 & 1.23 & 192 & 3.77 & 1.42 & 267.5 & 6.71 & 2.40 & 88.5 & \\
\hline Played a video game on his lunch break & 4.87 & 1.06 & 234.5 & 5.00 & 1.29 & 237 & 4.57 & 1.69 & 240.5 & 5.97 & 2.09 & 128 & \\
\hline Was very concerned $a b$ & & & & & & & & & & & & & \\
\hline & 4.85 & 1.89 & 237 & 4.92 & 1.52 & 312 & 5.20 & 1.61 & & & 2.23 & 206 & \\
\hline Told a & 4.85 & 1.46 & 237 & 3.92 & 1.38 & 252 & 4.26 & 1.20 & 213.5 & 4.74 & 2.47 & & \\
\hline Didn't u & & & & & & & & & & & & & \\
\hline Hope & 4.85 & 1.04 & 237 & 4.97 & 0.44 & 241 & 4.00 & 1.75 & 255 & 4.34 & 2.31 & 239.5 & \\
\hline Gets confused by the rules of chess & 4,80 & 0.86 & 239 & 5.05 & 0.58 & 231.5 & 4.34 & 1.21 & 248 & 5.57 & 2.28 & 152.5 & \\
\hline Attended aerobic exercise clas & & & & & & & & & & & & & \\
\hline & 4.77 & 1.78 & 240.5 & 4.97 & 1.50 & 241 & 4.60 & 1.17 & 239 & 6.49 & 2.09 & 104.5 & \\
\hline Scored & 4.77 & 1.48 & 240.5 & 4.95 & 0.78 & 246 & 4.03 & 1.10 & 25 & 5.97 & 2.26 & 128 & \\
\hline Boug & 4.74 & 1.31 & 243 & 5.16 & 0.65 & 214 & 5.34 & 1.81 & 20 & 8.97 & 2.02 & 6 & \\
\hline tract painting upside down & 4.74 & 1.97 & 243 & 4.65 & 0.86 & 278.5 & 4.29 & 0.96 & 25 & $4 . \mathrm{C}$ & 2.63 & & \\
\hline very Saturday & 4.74 & 1.16 & 243 & 5.40 & 1.28 & 180 & 5.03 & 1.54 & 227 & 3.91 & 2.69 & 271 & \\
\hline rules of soccer & 4.72 & 1.23 & 245 & 4.97 & 0.83 & 241 & 3.77 & 1.48 & 267.5 & 4.80 & 1.86 & 200.5 & \\
\hline woman's dress & 4.69 & 0.83 & 246.5 & 4.92 & 0.68 & 252 & 4.91 & 0.89 & 231 & 6.69 & 1.94 & 90 & \\
\hline & & & & & & & & & & & & & \\
\hline & 4.69 & 1.24 & 246.5 & 3.46 & 1.37 & 323 & 5.43 & 1.74 & 201 & 5.46 & 1.98 & 162.5 & \\
\hline Missed & 4.59 & 1.50 & 248 & 4.95 & 0.70 & 246 & 4.11 & 1.2 & 25 & 99 & 2.29 & & \\
\hline Misspelled several words in letters to friends & 4.46 & 1.00 & 249 & 5.05 & 0.74 & 231.5 & 4.09 & 0.89 & 253 & 6.43 & 2.39 & 108.5 & \\
\hline Confused the name of a famous art & & & & & & & & & & & & & \\
\hline & 4.41 & 1.09 & & 4.89 & 0.57 & 256 & 4.49 & 1.12 & & 4.51 & 2.80 & & \\
\hline llow a recipe for meat loaf & 4.41 & 1.19 & 250.5 & 4.95 & 0.70 & 246 & 3.51 & 1.22 & 278.5 & 4.46 & 1.88 & & \\
\hline ut enjoys comics & 4.39 & 1.71 & 252 & 4.95 & 1.05 & 246 & 4.31 & 1.59 & 249 & 5.60 & 2.17 & 149 & \\
\hline eps only 5 hours a night & 4.36 & 1.16 & 253 & 5.05 & 0.82 & & 4.77 & 1.19 & 238 & 5.6 & 1.97 & 147.5 & \\
\hline om a couple & 4.26 & 1.41 & 254 & 4.51 & 1.76 & 285.5 & 4.49 & 1.84 & 243.5 & 4.66 & 1.89 & 213 & \\
\hline Has $d$ & 4.23 & 1.33 & 255.5 & 4.95 & 0.78 & 246 & 3.74 & 1.38 & 269.5 & 4.77 & 1.85 & 20 & \\
\hline ding Time magazine & 4.23 & 0.93 & 255.5 & 4.92 & 0.68 & 252 & 3.88 & 1.02 & 260 & 4.43 & 1.88 & 232 & \\
\hline Did $p$ & 4.21 & 1.36 & 257.5 & 4.86 & 0.89 & 257.5 & 3.43 & 1.17 & & 4.83 & 1.93 & & \\
\hline Has & 4.21 & 1.5 & 257.5 & 4.92 & 0.80 & 252 & 3.80 & 0.90 & 26 & 4.1 & 1.97 & & \\
\hline Did not vote in the presidenti & 4.18 & 1.78 & 259 & 4.00 & 1.51 & 310 & 3.31 & 1.76 & 291.5 & 5.20 & 2.54 & 176 & \\
\hline $\begin{array}{l}\text { Was confused by the instructions for a ne } \\
\text { popcorn popper }\end{array}$ & 4.13 & 1.51 & 261 & 4.92 & 0.89 & 252 & 3.34 & 1.86 & 289.5 & 4.69 & 2.01 & 210.5 & \\
\hline Dropped out of high school and got a & & & & & & & & & & & & & \\
\hline & 4.13 & 1.63 & 261 & 4.84 & 0.99 & 260.5 & 3.43 & 1.70 & 284.5 & 4.40 & 2.19 & 235 & 5 \\
\hline Has difficulty using the card catalogue in & & & & & & & & & & & & & \\
\hline & & 1.24 & 261 & 4.73 & 0.73 & 272 & & 1.01 & & 4.26 & 2.36 & & \\
\hline incorrect grammar & 4.10 & 1.07 & 263 & 4.81 & 0.97 & 264 & 3.83 & 1.48 & 261 & 6.60 & 2.52 & 92.5 & \\
\hline & & & & & & & & & & & & & \\
\hline & & 1.14 & & 4.84 & 0.73 & & 3.46 & 1.27 & & & 2.50 & & \\
\hline Picked up several hitch-hikers & 4.08 & 2.32 & 265 & 6.00 & 2.06 & 94 & 2.03 & 1.50 & 375.5 & 2.66 & 1.94 & 351.5 & \\
\hline $\begin{array}{l}\text { Was unable to name the Vice President of } \\
\text { the U.S. }\end{array}$ & 4.05 & 1.41 & 266 & 4.92 & 0.55 & 252 & 3.26 & 2.13 & 294.5 & 4.40 & 2.79 & 235 & \\
\hline $\begin{array}{l}\text { Made a mathematical error } \\
\text { tax return }\end{array}$ & 4.00 & 1.49 & 267.5 & 5.05 & 0.58 & 231.5 & 4.51 & 1.10 & 242 & 6.74 & 2.16 & 86.5 & \\
\hline & & & & & & & & & & & & & \\
\hline & 4.00 & 1.40 & 267.5 & 3.49 & 1.22 & 322 & 4.94 & 1.59 & 230 & 3.34 & 2.10 & 312 & \\
\hline
\end{tabular}


APPENDIX (Continued)

\begin{tabular}{|c|c|c|c|c|c|c|c|c|c|c|c|c|c|}
\hline \multirow[b]{3}{*}{ Behavior } & \multicolumn{12}{|c|}{ Ratings } & \multirow[b]{3}{*}{ Cluster } \\
\hline & \multicolumn{3}{|c|}{ Goodness } & \multicolumn{3}{|c|}{ Kindness } & \multicolumn{3}{|c|}{ Intelligence } & \multicolumn{3}{|c|}{ Normality } & \\
\hline & $M$ & $S D$ & Rank & $M$ & $S D$ & Rank & $M$ & $S D$ & Rank & $M$ & $S D$ & Rank & \\
\hline
\end{tabular}

Lost his shoes at a Japanese restaurant

Could not figure out how to use an adding machine

$\begin{array}{llllll}3.97 & 1.20 & 269 & 4.27 & 1.48 & 300\end{array}$

$\begin{array}{lllllll}3.23 & 1.09 & 296.5 & 2.97 & 2.42 & 333.5 & 5\end{array}$

Could not remember his social security number

Mispronounces common words during

conversations

Measured his biceps every week

Forgot the name of his previous employer during an interview

Failed to follow the maintenance schedule

on his car

Forgot to tum the lights out in his car

Does not know how to cook a breakfast

$\begin{array}{lllllllllllll}3.92 & 1.51 & 270 & 4.68 & 0.94 & 276 & 3.89 & 1.78 & 259 & 3.46 & 1.46 & 302.5 & 5\end{array}$

$\begin{array}{lllllllllllll}3.90 & 1.47 & 271 & 4.73 & 0.61 & 272 & 3.31 & 1.39 & 291.5 & 3.34 & 2.51 & 312 & 5\end{array}$

$\begin{array}{lllllllllllll}3.87 & 1.24 & 272.5 & 4.81 & 0.57 & 264 & 3.60 & 1.14 & 275.5 & 4.03 & 2.43 & 258.5 & 5\end{array}$

$\begin{array}{llllllllllllll}3.87 & 1.56 & 272.5 & 4.57 & 1.09 & 281.5 & 4.40 & 1.38 & 245.5 & 3.60 & 2.08 & 292 & 5\end{array}$

$\begin{array}{lllllllllllll}3.82 & 1.37 & 274 & 4.32 & 0.92 & 294.5 & 3.51 & 1.46 & 278.5 & 5.00 & 2.51 & 187 & 5\end{array}$

$\begin{array}{lllllllllllll}3.80 & 1.36 & 275.5 & 4.70 & 0.97 & 274 & 3.14 & 1.67 & 301 & 6.51 & 2.19 & 101 & 5\end{array}$

$\begin{array}{lllllllllllll}3.80 & 1.40 & 275.5 & 4.76 & 0.64 & 269.5 & 3.57 & 1.29 & 277 & 5.66 & 2.07 & 147.5 & 5\end{array}$

$\begin{array}{llllllllllllll}3.69 & 1.17 & 277.5 & 4.78 & 1.06 & 267 & 3.43 & 1.27 & 284.5 & 3.20 & 1.80 & 322.5 & 5\end{array}$

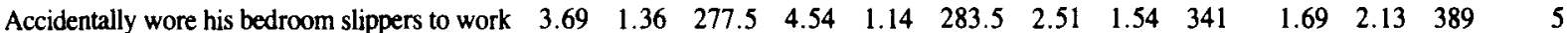

Could not remember the birthdays of his

family members

Rode his bicycle through broken glass and got a flat tire

Ran into a pole because he was not

watching where he was going

Always leaves the keys in his car

Ran out of gas twice on the same trip

Lost his wallet at the State Fair

Carries a switchblade knife

Took the wrong bus on 2 consecutive days

Failed the written driver's license exam

four times

Crammed for a math test when he was

having an English test

Did not know the zip code for his address

Was unable to write a business letter

Pulled his dog's tail

Ignored his old girlfriend at a party

Still gets lost on campus after being there

2 years

$\begin{array}{lllllllllllll}3.64 & 1.46 & 279 & 3.54 & 1.43 & 320.5 & 3.80 & 1.84 & 264 & 4.66 & 2.87 & 213 & 2\end{array}$

$\begin{array}{lllllllllllll}3.62 & 1.41 & 280.5 & 4.22 & 1.46 & 303.5 & 2.03 & 1.76 & 375.5 & 5.40 & 2.65 & 165 & 5\end{array}$

$\begin{array}{lllllllllllll}3.62 & 1.25 & 280.5 & 4.22 & 1.40 & 303.5 & 3.00 & 1.68 & 307.5 & 3.51 & 2.31 & 297.5 & 5\end{array}$

$\begin{array}{lllllllllllll}3.59 & 1.46 & 282.5 & 4.84 & 1.09 & 260.5 & 1.97 & 1.52 & 378 & 2.89 & 2.01 & 338.5 & 5\end{array}$

$\begin{array}{lllllllllllll}3.59 & 1.48 & 282.5 & 4.76 & 0.86 & 269.5 & 2.57 & 1.70 & 333.5 & 2.37 & 1.88 & 364.5 & 5\end{array}$

$\begin{array}{lllllllllllll}3.56 & 1.55 & 285.5 & 4.46 & 1.43 & 288 & 3.74 & 1.46 & 269.5 & 4.86 & 2.38 & 195 & 5\end{array}$

$\begin{array}{lllllllllllll}3.56 & 2.05 & 285.5 & 3.62 & 1.74 & 317 & 2.91 & 2.23 & 315.5 & 3.57 & 2.56 & 294 & 5\end{array}$

$\begin{array}{lllllllllllll}3.56 & 1.12 & 285.5 & 4.27 & 1.24 & 300 & 2.94 & 1.47 & 312.5 & 2.89 & 2.04 & 338.5 & 5\end{array}$

$\begin{array}{lllllllllllll}3.56 & 1.60 & 285.5 & 4.62 & 0.92 & 280 & 2.17 & 1.48 & 365 & 2.77 & 2.10 & 345 & 5\end{array}$

$\begin{array}{lllllllllllll}3.54 & 2.18 & 288 & 4.27 & 1.28 & 300 & 2.29 & 1.66 & 357.5 & 2.14 & 2.30 & 373 & 5\end{array}$

$\begin{array}{lllllllllllll}3.51 & 1.45 & 289 & 4.78 & 1.08 & 267 & 2.17 & 1.69 & 365 & 2.06 & 2.04 & 374.5 & 5\end{array}$

$\begin{array}{llllllllllllll}3.46 & 1.27 & 290 & 4.68 & 0.85 & 276 & 3.29 & 1.67 & 293 & 4.51 & 1.88 & 225 & 5\end{array}$

$\begin{array}{lllllllllllll}3.41 & 1.60 & 291 & 2.73 & 1.52 & 328 & 2.17 & 1.69 & 365 & 3.97 & 2.43 & 267 & 2\end{array}$

$\begin{array}{lllllllllllll}3.39 & 1.93 & 292 & 2.62 & 1.59 & 333 & 3.94 & 2.00 & 257.5 & 6.80 & 2.51 & 83 & 2\end{array}$

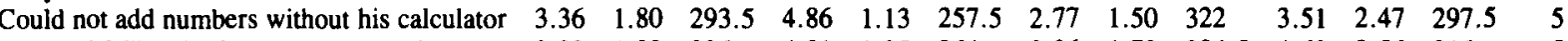

Watched MTV for hours every evening

Refused to let the neighborhood children play in his yard

Could not figure out how to get his car door unlocked

Flunked an aptitude test

Was irritated when a neighbor asked to borrow some milk

Always forgets where he put his wallet

Lost $\$ 700$ in a casino

$\begin{array}{lllllllllllll}3.33 & 1.99 & 296 & 4.81 & 1.35 & 264 & 3.26 & 1.79 & 294.5 & 4.63 & 2.56 & 216 & 5\end{array}$

$\begin{array}{lllllllllllll}3.33 & 1.87 & 296 & 2.62 & 1.50 & 333 & 4.40 & 1.65 & 245.5 & 4.63 & 1.68 & 216 & 2\end{array}$

$\begin{array}{lllllllllllll}3.33 & 1.55 & 296 & 4.68 & 0.94 & 276 & 2.37 & 2.22 & 352 & 2.20 & 1.80 & 372 & 5\end{array}$

$\begin{array}{lllllllllllll}3.31 & 1.73 & 298.5 & 4.49 & 1.17 & 287 & 3.20 & 1.71 & 299 & 3.74 & 2.02 & 285 & 5\end{array}$

$\begin{array}{lllllllllllll}3.31 & 1.42 & 298.5 & 2.62 & 1.46 & 333 & 3.94 & 1.83 & 257.5 & 3.71 & 2.18 & 287.5 & 2\end{array}$

$\begin{array}{llllllllllllll}3.28 & 1.55 & 301.5 & 4.78 & 6.58 & 267 & 3.46 & 1.79 & 281.5 & 4.97 & 1.72 & 189.5 & 5\end{array}$

$\begin{array}{lllllllllllll}3.28 & 1.76 & 301.5 & 4.73 & 1.22 & 272 & 2.14 & 1.91 & 369 & 4.40 & 2.50 & 235 & 5\end{array}$

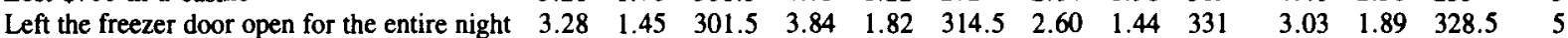

Could not do his daughter's fifth-grade homework problems

Hit the accelerator instead of the brake and hit another car

Didn't leave a tip at the restaurant

Forgot to take his book home to study the night before a test

Drove his car the wrong way down a oneway street

Locked himself in the basement when no one was home

$\begin{array}{lllllllllllll}3.28 & 1.50 & 301.5 & 4.35 & 1.34 & 292.5 & 2.80 & 1.62 & 319.5 & 2.97 & 2.55 & 333.5 & 5\end{array}$

$\begin{array}{llllllllllllll}3.26 & 1.39 & 304 & 4.32 & 1.47 & 294.5 & 3.37 & 2.00 & 288 & 4.57 & 2.05 & 219 & 5\end{array}$

$\begin{array}{lllllllllllll}3.23 & 1.35 & 306 & 2.62 & 1.38 & 333 & 3.80 & 1.37 & 264 & 5.09 & 1.85 & 181.5 & 2\end{array}$

$\begin{array}{lllllllllllll}3.23 & 1.65 & 306 & 4.14 & 1.58 & 306.5 & 2.86 & 2.09 & 317 & 4.74 & 2.02 & 206 & 5\end{array}$

$\begin{array}{lllllllllllll}3.23 & 1.40 & 306 & 3.95 & 1.63 & 311 & 2.66 & 1.92 & 328 & 4.20 & 2.36 & 245.5 & 5\end{array}$

$\begin{array}{lllllllllllll}3.18 & 1.65 & 308 & 4.40 & 1.46 & 290 & 2.69 & 1.71 & 326.5 & 2.63 & 2.02 & 353 & 5\end{array}$

$\begin{array}{lllllllllllll}3.15 & 1.53 & 309.5 & 2.40 & 1.40 & 342 & 3.49 & 1.62 & 280 & 4.31 & 1.97 & 241 & 2\end{array}$

$\begin{array}{llllllllllllll}3.15 & 1.53 & 309.5 & 4.57 & 1.50 & 281.5 & 2.54 & 1.29 & 336.5 & 3.49 & 1.87 & 300 & 5\end{array}$

$\begin{array}{lllllllllllll}3.10 & 1.64 & 312.5 & 4.65 & 1.40 & 278.5 & 3.34 & 1.53 & 289.5 & 5.57 & 2.09 & 152.5 & 5\end{array}$

$\begin{array}{lllllllllllll}3.10 & 1.86 & 312.5 & 2.95 & 1.65 & 325 & 3.80 & 1.98 & 264 & 5.54 & 2.19 & 157.5 & 2\end{array}$

$\begin{array}{llllllllllllll}3.10 & 2.20 & 312.5 & 2.43 & 1.59 & 339.5 & 3.80 & 1.64 & 264 & 3.97 & 1.69 & 267 & 2\end{array}$

$\begin{array}{lllllllllllll}3.10 & 1.92 & 312.5 & 2.68 & 1.90 & 330 & 3.71 & 2.28 & 271.5 & 2.94 & 1.63 & 335.5 & 2\end{array}$ 
APPENDIX (Continued)

\begin{tabular}{|c|c|c|c|c|c|c|c|c|c|c|c|c|c|}
\hline \multirow[b]{3}{*}{ Behavior } & \multicolumn{12}{|c|}{ Ratings } & \multirow[b]{3}{*}{ Cluster } \\
\hline & \multicolumn{3}{|c|}{ Goodness } & \multicolumn{3}{|c|}{ Kindness } & \multicolumn{3}{|c|}{ Intelligence } & \multicolumn{3}{|c|}{ Normality } & \\
\hline & $M$ & $S D$ & Rank & $M$ & $S D$ & Rank & $M$ & $S D$ & Rank & $M$ & $S D$ & Rank & \\
\hline
\end{tabular}

Let his dog go to the bathroom on the neighbor's yard

Laughed at a question asked by a classmate

Does not know the multiplication tables

Made a prank phone call

Failed to return a borrowed book for months

Scorched his face while shooting off fireworks

Went out of his way to tell strangers about his accomplishments

Walked into the street without checking for oncoming traffic

Refused to attend the funeral of a relative he disliked

Dropped his keys out the window of a moving car

Kept the windows open while running the air conditioner

Went swimming in $30^{\circ}$ weather

Flushed his house keys down the toilet

Forgot to dress well for his job interview

Ignored a neighbor's request to borrow some tools

Does not wear a coat in extremely cold weather

Left a gas station without removing the gas nozzle from his car

Left his windows open while washing his car

Heckled a woman speaking on human rights

Forgot to turn off the bathroom water,

flooding the house

"Bounced" several checks in 1 year

Laughed at a person who tripped on a curb and fell

Told a colleague in public that she should lose weight

Asked the same question in class three times

Spent money compulsively on items he didn't need

Forgot to turn the stove off before going on a trip

Refused to help a classmate with a homework assignment

Returned a birthday gift because it was too cheap

Loudly talked to a companion in a movie

Kicked a stray cat to get it to leave his yard

Got into a fight in a bar with an acquaintance

Took the neighbor's newspaper

Threw an acquaintance out of his home for disagreeing with him

Laughed at a little-league player who ran slowly

Considered himself to be an authority on all subjects

Started an argument with a coworker

Kicked his dog for eating cheese on the edge of the table

Lied to his girlfriend about not having other dates

Refused to help a child fix his bicycle

Refused to take phone messages for a roommate

Shoved a man who was passing out leaflets

Closed the elevator door before anyone else could get on

Ate all the food before anyone else could have any $\begin{array}{lll}3.08 & 1.42 & 316\end{array}$

$\begin{array}{lll}3.08 & 1.86 & 316\end{array}$

$\begin{array}{lll}3.08 & 1.61 & 316\end{array}$

$\begin{array}{lll}3.00 & 1.59 & 318\end{array}$

$\begin{array}{lll}2.97 & 1.48 & 320\end{array}$

$\begin{array}{lll}2.97 & 1.65 & 320\end{array}$

$2.97 \quad 1.65 \quad 320$

$\begin{array}{lll}2.60 & 1.69 & 336\end{array}$

$\begin{array}{llllll}3.71 & 2.12 & 271.5 & 5.11 & 2.39 & 179\end{array}$

$\begin{array}{llllllllll}2.43 & 1.48 & 339.5 & 2.94 & 2.01 & 312.5 & 4.83 & 2.12 & 197.5\end{array}$

$\begin{array}{lllllllll}4.51 & 1.14 & 285.5 & 2.77 & 1.85 & 322 & 2.69 & 1.95 & 349.5\end{array}$

$\begin{array}{llllllllll}2.92 & 1.48 & 326 & 2.77 & 1.96 & 322 & 5.94 & 2.29 & 131.5\end{array}$

2.92

$\begin{array}{lll}2.84 & 1.30 & 327\end{array}$

$2.60 \quad 1.22 \quad 331$

$\begin{array}{lll}3.66 & 2.40 & 290.5\end{array}$

3.891 .52 313

$\begin{array}{lll}3.63 & 1.40 & 274\end{array}$

$\begin{array}{lll}3.34 & 2.14 & 312\end{array}$

$\begin{array}{llllllllllll}2.92 & 1.58 & 322 & 4.35 & 1.32 & 292.5 & 1.86 & 1.80 & 381.5 & 4.11 & 2.27 & 250.5\end{array}$

$\begin{array}{lllllllllllll}2.87 & 1.64 & 323.5 & 2.40 & 2.17 & 342 & 3.20 & 1.81 & 299 & 3.77 & 1.97 & 281.5 & 2\end{array}$

$\begin{array}{lllllllllllll}2.87 & 2.12 & 323.5 & 4.27 & 1.35 & 300 & 2.63 & 2.03 & 329 & 1.06 & 1.73 & 397 & 5\end{array}$

$\begin{array}{lllllllllllll}2.85 & 1.50 & 326 & 3.84 & 1.46 & 314.5 & 2.46 & 2.16 & 347 & 2.83 & 1.96 & 342 & 5\end{array}$

$\begin{array}{lllllllllllll}2.85 & 1.91 & 326 & 4.30 & 1.35 & 296.5 & 2.09 & 2.12 & 373 & 1.51 & 1.48 & 392 & 5\end{array}$

$\begin{array}{lllllllllllll}2.85 & 1.63 & 326 & 4.40 & 1.34 & 290 & 1.69 & 1.73 & 391 & 1.23 & 1.40 & 396 & 5\end{array}$

$\begin{array}{lllllllllllll}2.82 & 1.36 & 328 & 4.03 & 1.44 & 309 & 2.23 & 1.14 & 360.5 & 2.40 & 1.90 & 362.5 & 5\end{array}$

$\begin{array}{llllllllllllll}2.74 & 1.31 & 329.5 & 2.46 & 1.17 & 338 & 3.60 & 1.77 & 275.5 & 4.03 & 2.01 & 258.5 & 2\end{array}$

$\begin{array}{lllllllllllll}2.74 & 1.65 & 329.5 & 4.30 & 1.47 & 296.5 & 1.91 & 1.44 & 379 & 2.60 & 2.20 & 354.5 & 5\end{array}$

$\begin{array}{lllllllllllll}2.69 & 1.49 & 331.5 & 4.14 & 1.44 & 306.5 & 2.20 & 2.00 & 362.5 & 3.03 & 2.02 & 328.5 & 5\end{array}$

$\begin{array}{lllllllllllll}2.69 & 1.75 & 331.5 & 4.40 & 1.54 & 290 & 1.40 & 1.50 & 395.5 & 2.31 & 2.30 & 366.5 & 5\end{array}$

$\begin{array}{lllllllllllll}2.67 & 1.51 & 333.5 & 2.16 & 1.56 & 350 & 2.71 & 1.66 & 324.5 & 4.71 & 2.19 & 208.5 & 2\end{array}$

$\begin{array}{lllllllllllll}2.67 & 1.49 & 333.5 & 3.62 & 1.60 & 317 & 2.14 & 2.00 & 369 & 1.94 & 1.77 & 380.5 & 5\end{array}$

$\begin{array}{llllllllllllll}2.64 & 1.68 & 335 & 3.54 & 1.71 & 320.5 & 2.29 & 1.58 & 357.5 & 4.11 & 2.61 & 250.5 & 5\end{array}$

$\begin{array}{lllllllllllll}2.62 & 1.84 & 337 & 1.84 & 1.38 & 362.5 & 2.83 & 1.87 & 318 & 5.74 & 2.58 & 140.5 & 2\end{array}$

$\begin{array}{lllllllllllll}2.62 & 1.86 & 337 & 2.00 & 1.62 & 357 & 2.37 & 1.61 & 352 & 2.29 & 1.51 & 368.5 & 2\end{array}$

$\begin{array}{lllllllllllll}2.62 & 1.68 & 337 & 3.60 & 1.50 & 319 & 1.83 & 1.25 & 384 & 1.83 & 1.72 & 385 & 5\end{array}$

$\begin{array}{lllllllllllll}2.59 & 1.35 & 339 & 4.27 & 1.61 & 300 & 2.71 & 1.74 & 324.5 & 5.34 & 2.06 & 168 & 5\end{array}$

$\begin{array}{lllllllllllll}2.56 & 1.73 & 340 & 4.05 & 1.81 & 308 & 2.31 & 2.26 & 355 & 2.89 & 2.48 & 338.5 & 5\end{array}$

$\begin{array}{lllllllllllll}2.54 & 1.60 & 341 & 2.22 & 1.57 & 349 & 3.11 & 1.75 & 303 & 3.23 & 1.77 & 320.5 & 2\end{array}$

$\begin{array}{lllllllllllll}2.51 & 1.86 & 342 & 1.62 & 1.46 & 377 & 2.94 & 2.01 & 312.5 & 2.71 & 2.40 & 348 & 2\end{array}$

$\begin{array}{lllllllllllll}2.49 & 1.59 & 343.5 & 2.35 & 1.40 & 346 & 2.69 & 1.73 & 326.5 & 4.54 & 2.31 & 222 & 2\end{array}$

$\begin{array}{lllllllllllll}2.49 & 2.02 & 343.5 & 1.22 & 1.68 & 391.5 & 2.43 & 2.02 & 348 & 4.06 & 2.27 & 254.5 & 2\end{array}$

$\begin{array}{lllllllllllll}2.46 & 1.34 & 346 & 2.70 & 1.65 & 329 & 2.94 & 2.13 & 312.5 & 4.46 & 2.01 & 229.5 & 2\end{array}$

$\begin{array}{lllllllllllll}2.46 & 1.90 & 346 & 2.51 & 1.73 & 337 & 3.20 & 1.55 & 299 & 3.43 & 1.93 & 304.5 & 2\end{array}$

$\begin{array}{lllllllllllll}2.46 & 2.09 & 346 & 2.32 & 2.12 & 347 & 2.37 & 1.80 & 352 & 2.74 & 1.56 & 347 & 2\end{array}$

$\begin{array}{lllllllllllll}2.44 & 1.86 & 348.5 & 1.97 & 1.57 & 359 & 3.03 & 1.92 & 306 & 3.66 & 2.16 & 290.5 & 2\end{array}$

$\begin{array}{lllllllllllll}2.44 & 2.00 & 348.5 & 3.32 & 1.83 & 324 & 3.06 & 2.01 & 305 & 3.37 & 2.45 & 308 & 5\end{array}$

$\begin{array}{lllllllllllll}2.41 & 1.41 & 350.5 & 2.62 & 1.21 & 333 & 3.40 & 1.70 & 287 & 6.29 & 2.26 & 114 & 2\end{array}$

$\begin{array}{lllllllllllll}2.41 & 1.86 & 350.5 & 2.38 & 1.70 & 344.5 & 3.43 & 2.38 & 284.5 & 3.91 & 1.95 & 271 & 2\end{array}$

$\begin{array}{lllllllllllll}2.39 & 2.65 & 353 & 2.03 & 1.52 & 355.5 & 2.20 & 2.03 & 362.5 & 6.03 & 2.11 & 124 & 2\end{array}$

$\begin{array}{lllllllllllll}2.39 & 1.55 & 353 & 2.27 & 1.61 & 348 & 3.23 & 1.66 & 296.5 & 3.37 & 1.65 & 308 & 2\end{array}$

$\begin{array}{lllllllllllll}2.39 & 1.98 & 353 & 1.65 & 1.38 & 373.5 & 2.54 & 1.56 & 336.5 & 2.31 & 1.84 & 366.5 & 2\end{array}$

$\begin{array}{lllllllllllll}2.31 & 1.64 & 355 & 1.65 & 1.25 & 373.5 & 2.37 & 1.54 & 352 & 3.00 & 1.93 & 331.5 & 2\end{array}$

$\begin{array}{lllllllllllll}2.23 & 1.80 & 356 & 2.05 & 1.79 & 353 & 3.11 & 1.78 & 303 & 3.54 & 2.54 & 296 & 2\end{array}$

$\begin{array}{lllllllllllll}2.21 & 1.91 & 357 & 2.40 & 1.89 & 342 & 3.66 & 1.81 & 273 & 4.00 & 2.09 & 263.5 & 2\end{array}$ 
APPENDIX (Continued)

\begin{tabular}{|c|c|c|c|c|c|c|c|c|c|c|c|c|c|}
\hline \multirow[b]{3}{*}{ Behavior } & \multicolumn{12}{|c|}{ Ratings } & \multirow[b]{3}{*}{ Cluster } \\
\hline & \multicolumn{3}{|c|}{ Goodness } & \multicolumn{3}{|c|}{ Kindness } & \multicolumn{3}{|c|}{ Intelligence } & \multicolumn{3}{|c|}{ Normality } & \\
\hline & $M$ & $S D$ & Rank & $M$ & $S D$ & Rank & $M$ & $S D$ & Rank & $M$ & $S D$ & Rank & \\
\hline
\end{tabular}

Ignored a new person in the office for several weeks

Refused to loan class notes to a friend who

had been ill

Beat up someone smaller than him

$\begin{array}{lllllllllllll}2.18 & 1.73 & 358.5 & 2.05 & 1.39 & 353 & 2.29 & 1.56 & 357.5 & 3.80 & 2.34 & 278.5 & 2\end{array}$

Stole into a parking place while someone

else was backing in

Slammed the door in the face of a charity collector

Ridicules people behind their backs

Used bad language in front of children

Could not spell his name correctly

Scratched a stranger's car with his key

Stole Halloween candy

Refused to give his seat on the bus to an elderly woman

Provoked a stranger into a fight

Used stolen food stamps

Put sugar in his friend's gas tank

Hit a car and left the scene of the accident

Pushed into the front of a line at a theater

Loudly criticized the art at a community children's art show

Put a roach down another person's shirt

Tripped someone in the hall

Siphoned gas from a car parked on the street

Started a false rumor about someone

$\begin{array}{lllllllllllll}2.18 & 1.57 & 358.5 & 1.84 & 1.88 & 362.5 & 2.97 & 1.69 & 309.5 & 2.06 & 1.55 & 374.5 & 2 \\ 2.15 & 1.65 & 360.5 & 2.05 & 1.90 & 353 & 2.51 & 1.79 & 341 & 4.77 & 2.68 & 203 & 2\end{array}$

$\begin{array}{lllllllllllll}2.15 & 1.51 & 360.5 & 1.54 & 1.39 & 380 & 2.14 & 1.72 & 369 & 3.49 & 2.15 & 300 & 2\end{array}$

$\begin{array}{lllllllllllll}2.13 & 1.77 & 362 & 1.81 & 1.39 & 366 & 2.14 & 1.83 & 369 & 3.57 & 2.06 & 294 & 2\end{array}$

$\begin{array}{lllllllllllll}2.05 & 1.89 & 363 & 1.57 & 1.52 & 378.5 & 2.80 & 1.83 & 319.5 & 4.06 & 2.40 & 254.5 & 2\end{array}$

$\begin{array}{lllllllllllll}2.00 & 1.99 & 364.5 & 1.68 & 1.92 & 371 & 2.54 & 1.65 & 336.5 & 5.97 & 2.35 & 128 & 2\end{array}$

$\begin{array}{lllllllllllll}2.00 & 1.43 & 364.5 & 2.38 & 1.60 & 344.5 & 3.11 & 2.06 & 303 & 4.51 & 2.33 & 225 & 2\end{array}$

$\begin{array}{lllllllllllll}2.00 & 1.89 & 366 & 4.16 & 1.61 & 305 & 1.00 & 1.26 & 400 & 0.86 & 1.80 & 399 & 5\end{array}$

$\begin{array}{lllllllllllll}1.95 & 1.56 & 367 & 1.84 & 1.74 & 362.5 & 2.40 & 2.16 & 349 & 2.86 & 2.17 & 341 & 2\end{array}$

$\begin{array}{lllllllllllll}1.92 & 1.63 & 368 & 1.97 & 1.61 & 359 & 2.51 & 1.80 & 341 & 3.37 & 2.33 & 308 & 2\end{array}$

$\begin{array}{lllllllllllll}1.90 & 1.65 & 369 & 1.78 & 2.18 & 368.5 & 2.03 & 1.74 & 375.5 & 2.80 & 2.45 & 343 & 2\end{array}$

$\begin{array}{lllllllllllll}1.87 & 2.11 & 371 & 1.78 & 1.51 & 368.5 & 1.83 & 1.60 & 384 & 3.06 & 2.20 & 325.5 & 2\end{array}$

$\begin{array}{lllllllllllll}1.87 & 1.75 & 371 & 1.81 & 1.58 & 366 & 1.77 & 1.77 & 386.5 & 2.23 & 2.10 & 370.5 & 2\end{array}$

$\begin{array}{lllllllllllll}1.87 & 1.40 & 371 & 1.81 & 1.87 & 366 & 2.11 & 2.25 & 372.5 & 1.34 & 1.47 & 395 & 2\end{array}$

$\begin{array}{lllllllllllll}1.85 & 2.18 & 374 & 1.16 & 1.77 & 394 & 1.49 & 1.65 & 393 & 3.77 & 2.22 & 281.5 & 2\end{array}$

$\begin{array}{lllllllllllll}1.85 & 1.89 & 374 & 1.49 & 1.32 & 382.5 & 1.86 & 1.46 & 381.5 & 3.43 & 2.32 & 304.5 & 2\end{array}$

$\begin{array}{lllllllllllll}1.85 & 1.83 & 374 & 2.03 & 1.61 & 355.5 & 3.00 & 2.09 & 307.5 & 1.71 & 1.66 & 388 & 2\end{array}$

$\begin{array}{llllllllllllll}1.80 & 1.72 & 376 & 1.65 & 1.51 & 373.5 & 2.49 & 1.80 & 345 & 1.66 & 1.66 & 390 & 2\end{array}$

$\begin{array}{lllllllllllll}1.72 & 1.50 & 377 & 1.84 & 1.68 & 362.5 & 2.37 & 1.66 & 352 & 3.11 & 2.30 & 324 & 2\end{array}$

$\begin{array}{lllllllllllll}1.62 & 1.68 & 378 & 1.65 & 1.25 & 373.5 & 2.54 & 2.05 & 336.5 & 2.43 & 2.20 & 361 & 2\end{array}$

$\begin{array}{lllllllllllll}1.59 & 1.62 & 379.5 & 1.27 & 1.33 & 389.5 & 2.49 & 1.74 & 345 & 4.80 & 2.27 & 200.5 & 2\end{array}$

$\begin{array}{lllllllllllll}1.59 & 1.71 & 379.5 & 0.92 & 0.86 & 397 & 1.74 & 1.65 & 389 & 4.03 & 2.23 & 258.5 & 2\end{array}$

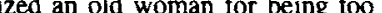

Defaced a large rock with spray paint in a public park

Spat off the balcony at the theater

$\begin{array}{lllllllllllll}1.56 & 1.40 & 381 & 2.08 & 1.44 & 351 & 1.77 & 1.72 & 386.5 & 2.77 & 1.77 & 345 & 2\end{array}$

$\begin{array}{lllllllllllll}1.51 & 1.95 & 382 & 1.97 & 1.66 & 359 & 2.03 & 1.50 & 375.5 & 3.00 & 1.96 & 331.5 & 2\end{array}$

Ignored a woman's cry for help in lifting a baby carriage

Made an obscene gesture at an old lady

Smoked in a no-smoking section even though others complained

Broke a friend's album and neither apologized or replaced it

Shot a songbird with his .22 caliber rifle

Hit a dog and drove away without notifying the owner

Continually berated his wife in public

Refused to hold the door for a man in a wheelchair

Tricked a housewife into paying for a nonexistent magazine

Insulted a stranger by making a racial slur

Pulled the seat out from underneath somebody

Was arrested on suspicion of child beating

Turned in someone else's project under his own name

Sells drugs to high school students

Stole money and jewelry from the relatives he was living with

Ridiculed a handicapped child by making fun of him

Attempted to rape a woman who was walking down a dark street

$\begin{array}{llllllllllll}1.41 & 1.33 & 384.5 & 1.57 & 1.80 & 378.5 & 1.83 & 1.69 & 384 & 2.00 & 1.59 & 378\end{array}$

$\begin{array}{lllllllllllll}1.41 & 1.62 & 384.5 & 1.51 & 1.66 & 381 & 2.57 & 1.84 & 333.5 & 1.91 & 2.06 & 383 & 2\end{array}$

$\begin{array}{lllllllllllll}1.39 & 1.66 & 386 & 1.49 & 1.17 & 382.5 & 1.26 & 1.46 & 398 & 2.77 & 1.90 & 345 & 2\end{array}$

$\begin{array}{lllllllllllll}1.36 & 1.56 & 387 & 1.76 & 1.92 & 370 & 2.49 & 1.96 & 345 & 2.54 & 2.17 & 357 & 2\end{array}$

$\begin{array}{lllllllllllll}1.33 & 1.58 & 388 & 1.32 & 1.97 & 387.5 & 2.14 & 2.06 & 369 & 2.03 & 2.23 & 376 & 2\end{array}$

$\begin{array}{lllllllllllll}1.31 & 1.51 & 389 & 1.22 & 1.18 & 391.5 & 2.60 & 1.68 & 331 & 3.03 & 1.71 & 328.5 & 2\end{array}$

$\begin{array}{lllllllllllll}1.31 & 1.92 & 390 & 1.27 & 1.33 & 389.5 & 1.74 & 2.25 & 389 & 2.00 & 1.75 & 378 & 2\end{array}$

$\begin{array}{lllllllllllll}1.31 & 1.76 & 390 & 1.00 & 1.27 & 396 & 1.46 & 1.46 & 394 & 1.77 & 1.73 & 386.5 & 2\end{array}$

$\begin{array}{lllllllllllll}1.28 & 1.54 & 392 & 1.35 & 1.78 & 385.5 & 2.91 & 2.63 & 315.5 & 2.29 & 1.87 & 368.5 & 2\end{array}$

$\begin{array}{lllllllllllll}1.23 & 1.29 & 393.5 & 1.14 & 1.11 & 395 & 2.23 & 2.18 & 360.5 & 4.91 & 2.58 & 192 & 2\end{array}$

$\begin{array}{lllllllllllll}1.23 & 1.25 & 393.5 & 1.38 & 1.38 & 384 & 1.89 & 1.73 & 380 & 3.34 & 2.41 & 312 & 2\end{array}$

$\begin{array}{lllllllllllll}1.18 & 1.79 & 395 & 1.64 & 1.75 & 376 & 2.51 & 2.08 & 341 & 1.91 & 2.11 & 383 & 2\end{array}$

$\begin{array}{lllllllllllll}1.15 & 1.51 & 396 & 1.32 & 1.23 & 387.5 & 1.74 & 1.82 & 389 & 3.03 & 2.26 & 328.5 & 2\end{array}$

$\begin{array}{lllllllllllll}0.92 & 1.66 & 397 & 1.35 & 1.58 & 385.5 & 1.14 & 1.67 & 399 & 2.69 & 2.41 & 349.5 & 2\end{array}$

$\begin{array}{lllllllllllll}0.74 & 1.35 & 398 & 0.86 & 1.08 & 398 & 1.54 & 1.76 & 392 & 1.40 & 1.27 & 394 & 2\end{array}$

$\begin{array}{lllllllllllll}0.64 & 0.96 & 399 & 0.73 & 1.15 & 399 & 1.40 & 2.17 & 395.5 & 1.94 & 1.98 & 380.5 & 2\end{array}$

$\begin{array}{lllllllllllll}0.51 & 1.57 & 400 & 0.24 & 0.50 & 400 & 1.37 & 2.00 & 397 & 1.00 & 1.24 & 398 & 2\end{array}$

Note-Rank refers to the position of the mean rating of the behavior relative to the mean ratings of the other behaviors for the same trait dimension.

(Manuscript received January 13, 1989;

revision accepted for publication September 20, 1989.) 